\title{
BARYCENTRIC STRAIGHTENING AND BOUNDED COHOMOLOGY
}

\author{
JEAN-FRANÇOIS LAFONT $^{\dagger}$ AND SHI WANG
}

\begin{abstract}
AвstRact. We study the barycentric straightening of simplices in higher rank irreducible symmetric spaces of non-compact type. We show that, for an $n$-dimensional symmetric space of rank $r \geq 2$ (excluding $S L(3, \mathbb{R}) / S O(3)$ and $S L(4, \mathbb{R}) / S O(4))$, the $p$-Jacobian has uniformly bounded norm, provided $p \geq n-r+2$. As a consequence, for the corresponding non-compact, connected, semisimple real Lie group $G$, in degrees $p \geq n-r+2$, every degree $p$ cohomology class has a bounded representative. This answers Dupont's problem in small codimension. We also give examples of symmetric spaces where the barycentrically straightened simplices of dimension $n-r$ have unbounded volume, showing that the range in which we obtain boundedness of the $p$-Jacobian is very close to optimal.
\end{abstract}

\section{INTRODUCTION}

When studying the bounded cohomology of groups, an important theme is the comparison map from bounded cohomology to ordinary cohomology. In the context of non-compact, connected, semisimple Lie groups, Dupont raised the question of whether this comparison map is always surjective [10] (see also Monod's ICM address [17, Problem A'], and [4, Conjecture 18.1]). Properties of these Lie groups $G$ are closely related to properties of the corresponding non-positively curved symmetric space $X=G / K$. Geometric methods on the space $X$ can often be used to recover information about the Lie group $G$. This philosophy was used by Lafont and Schmidt [16] to show that the comparison map is surjective in degree $\operatorname{dim}(X)$. In the present paper, we extend this result to smaller degrees, and show:

Main Theorem. Let $X=G / K$ be an $n$-dimensional irreducible symmetric space of non-compact type of $\operatorname{rank} r=\operatorname{rank}(X) \geq 2$, excluding $S L(3, \mathbb{R}) / S O(3)$ and $S L(4, \mathbb{R}) / S O(4)$, and $\Gamma$ a cocompact torsion-free lattice in $G$. Then the comparison maps $\eta: H_{c, b}^{*}(G, \mathbb{R}) \rightarrow H_{c}^{*}(G, \mathbb{R})$ and $\eta^{\prime}: H_{b}^{*}(\Gamma, \mathbb{R}) \rightarrow H^{*}(\Gamma, \mathbb{R})$ are both surjective in all degrees $* \geq n-r+2$.

The idea of the proof is similar to that in [16]. One defines a barycentric straightening of simplices in $X$, and uses it to construct bounded cocycles representing any given cohomology class. These cocycles are obtained by integrating a suitable differential form on various straightened simplices. Since the differential form has bounded norm, the key step is to show that the Jacobian of the straightened simplex is uniformly controlled (independent of the simplex or the point in it). Showing this later property requires some work,

1991 Mathematics Subject Classification. 57T10 (primary), 53C35 (secondary).

Key words and phrases. Barycenter method, bounded cohomology, semisimple Lie group, Dupont's problem.

$\dagger$ The work of the first author is partially supported by the NSF, under grants DMS-1510640, DMS-1812028. 
and is done in Sections 3 and 4 (following the general approach of Connell and Farb [6], [7]). The proof of the Main Theorem is then given in Section 5 .

Remark. For the various families of higher rank symmetric spaces, the dimension grows roughly quadratically in the rank. Our Main Theorem thus answers Dupont's question for continuous cohomology classes in degree close to the dimension of the symmetric space. Prior results on this problem include some work on the degree two case (Domic and Toledo [8], as well as Clerk and Orsted [5]) as well as the top-degree case (Lafont and Schmidt [16]). In his seminal paper on the subject, Gromov showed that characteristic classes of flat bundles are bounded classes [13]. Using Gromov's result, Hartnick and Ott [14] were able to obtain complete answers for several specific classes of Lie groups (e.g. of Hermitian type, as well as some other cases).

The recently posted preprint [15] of Inkang Kim and Sungwoon Kim uses similar methods to obtain uniform control of the Jacobian in codimension one. Their paper also contains a wealth of other applications, which we have not pursued in the present paper. On the other hand, their results do not produce any new bounded cohomology classes (since in the higher rank case, the codimension one continuous cohomology always vanishes).

\section{Acknowledgments}

We would like to thank Michelle Bucher-Karlsson, Marc Burger, Chris Connell, Tobias Hartnick, Clara Löh, Ben McReynolds, and Roman Sauer for their helpful comments.

\section{Preliminaries}

2.1. Symmetric spaces of non-compact type. In this section, we give a quick review of some results on symmetric spaces of non-compact type; for more details, we refer the reader to Eberlein's book [11]. Let $X=G / K$ be a symmetric space of non-compact type, where $G$ is semisimple and $K$ is a maximal compact subgroup of $G$. Geometrically $G$ can be identified with $\operatorname{Isom}_{0}(X)$, the connected component of the isometry group of $X$ that contains the identity, and $K=\operatorname{Stab}_{p}(G)$ for some $p \in X$. Fixing a basepoint $p \in X$, we have a Cartan decomposition $\mathfrak{g}=\mathfrak{f}+\mathfrak{p}$ of the Lie algebra $\mathfrak{g}$ of $G$, where $\mathfrak{f}$ is the Lie algebra of $K$, and $\mathfrak{p}$ can be isometrically identified with $T_{p} X$ using the Killing form. Let $\mathfrak{a} \subseteq \mathfrak{p}$ be a maximal abelian subalgebra of $\mathfrak{p}$. We can identify $\mathfrak{a}$ with the tangent space of a flat $\mathcal{F}$ at $p$ - that is to say, an isometrically embedded Euclidean space $\mathbb{R}^{r} \subseteq X$, where $r$ is the rank of $X$. Given any vector $v \in T_{p} X$, there exists a flat $\mathcal{F}$ that is tangent to $v$. We say $v$ is regular if such a flat is unique, and singular otherwise.

Now let $v \in \mathfrak{p}$ be a regular vector. This direction defines a point $v(\infty)$ on the visual boundary $\partial X$ of $X$. $G$ acts on the visual boundary $\partial X$. The orbit set $G v(\infty)=\partial_{F} X \subseteq \partial X$ is called a Furstenberg boundary of $X$. Since both $G$ and $K$ act transitively on $\partial_{F} X, \partial_{F} X$ is compact. In fact, a point stabilizer for the $G$-action on $\partial_{F} X$ is a minimal parabolic subgroup $P$, so we can also identify $\partial_{F} X$ with the quotient $G / P$. In the rest of this paper, we will use a specific realization of the Furstenberg boundary - the one given by choosing the regular vector $v$ to point towards a barycenter of a Weyl chamber in the flat. 
For each element $\alpha$ in the dual space $\mathfrak{a}^{*}$ of $\mathfrak{a}$, we define $\mathfrak{g}_{\alpha}=\{Y \in \mathfrak{g} \mid[A, Y]=\alpha(A) Y$ for all $A \in \mathfrak{a}\}$. We call $\alpha$ a root if $\mathfrak{g}_{\alpha}$ is nontrivial, and in such case we call $\mathfrak{g}_{\alpha}$ the root space of $\alpha$. We denote the finite set of roots $\Lambda$, and we have the following root space decomposition

$$
\mathfrak{g}=\mathfrak{g}_{0} \oplus \bigoplus_{\alpha \in \Lambda} \mathfrak{g}_{\alpha}
$$

where $\mathfrak{g}_{0}=\{Y \in \mathfrak{g} \mid[A, Y]=0$ for all $A \in \mathfrak{a}\}$, and the direct sum is orthogonal with respect to the canonical inner product on $\mathfrak{g}$.

Let $\theta$ be the Cartan involution at the point $p$. Then $\theta$ is an involution on $\mathfrak{g}$, which acts by $I$ on $\mathfrak{f}$ and $-I$ on $\mathfrak{p}$, hence it preserves Lie bracket. We can define $\mathfrak{f}_{\alpha}=(I+\theta) \mathfrak{g}_{\alpha} \subseteq \mathfrak{f}$, and $\mathfrak{p}_{\alpha}=(I-\theta) \mathfrak{g}_{\alpha} \subseteq \mathfrak{p}$, with the following properties:

Proposition 2.1. [11, Proposition 2.14.2] (1) $I+\theta: \mathfrak{g}_{\alpha} \rightarrow \mathfrak{f}_{\alpha}$ and $I-\theta: \mathfrak{g}_{\alpha} \rightarrow \mathfrak{p}_{\alpha}$ are linear isomorphisms. Hence $\operatorname{dim}\left(\mathfrak{f}_{\alpha}\right)=\operatorname{dim}\left(\mathfrak{g}_{\alpha}\right)=\operatorname{dim}\left(\mathfrak{p}_{\alpha}\right)$.

(2) $\mathfrak{f}_{\alpha}=\mathfrak{\mathfrak { F }}_{-\alpha}$ and $\mathfrak{p}_{\alpha}=\mathfrak{p}_{-\alpha}$ for all $\alpha \in \Lambda$, and $\mathfrak{\mathfrak { F }}_{\alpha} \oplus \mathfrak{p}_{\alpha}=\mathfrak{g}_{\alpha} \oplus \mathfrak{g}_{-\alpha}$.

(3) $\mathfrak{\mathfrak { f }}=\mathfrak{f}_{0} \oplus \bigoplus_{\alpha \in \Lambda^{+}} \mathfrak{f}_{\alpha}$ and $\mathfrak{p}=\mathfrak{a} \oplus \bigoplus_{\alpha \in \Lambda^{+}} \mathfrak{p}_{\alpha}$, where $\mathfrak{f}_{0}=\mathfrak{g}_{0} \cap \mathfrak{F}$, and $\Lambda^{+}$is the set of positive roots.

Remark. Since $\mathfrak{p}_{\alpha}=\left(\mathfrak{g}_{\alpha}+\mathfrak{g}_{-\alpha}\right) \cap \mathfrak{p}$, the direct sum of $\mathfrak{p}$ in (3) of Proposition 2.1 is also orthogonal with respect to the canonical inner product on $\mathfrak{p}$.

We now analyze the adjoint action of $\mathfrak{f}$ on $\mathfrak{a}$. Let $u \in \mathfrak{f}_{\alpha}$ and $v \in \mathfrak{a}$, we can write $u$ as $(I+\theta) w$ where $w \in \mathfrak{g}_{\alpha}$, hence we have

$$
\begin{aligned}
{[u, v] } & =[(I+\theta) w, v]=[w, v]+[\theta w, v]=-\alpha(v) w+\theta[w,-v] \\
& =-\alpha(v) w+\theta(\alpha(v) w)=-\alpha(v)(I-\theta)(w) \\
& =-\alpha(v)(I-\theta)(I+\theta)^{-1} u
\end{aligned}
$$

This gives the following proposition.

Proposition 2.2. Let $\alpha$ be a root. The adjoint action of $\mathfrak{f}_{\alpha}$ on $\mathfrak{a}$ is given by

$$
[u, v]=-\alpha(v)(I-\theta)(I+\theta)^{-1} u
$$

for any $u \in \mathfrak{f}_{\alpha}$ and $v \in \mathfrak{a}$. In particular, $\mathfrak{\mathfrak { f }}_{\alpha}$ maps $v$ into $\mathfrak{p}_{\alpha}$.

Assume $v \in \mathfrak{a} \subseteq T_{x} X$ is inside a fixed flat through $x$, and let $K_{v}$ be the stabilizer of $v$ in $K$. Then the space $K_{v} \mathfrak{a}$ is the tangent space of the union of all flats that goes through $v$. Equivalently, it is the union of all vectors that are parallel to $v$, hence it can be identified with $\mathfrak{a} \oplus \bigoplus_{\alpha \in \Lambda^{+}, \alpha(v)=0} \mathfrak{p}_{\alpha}$. In particular, if $v$ is regular, then the space is just $\mathfrak{a}$. Moreover, if we denote by $\mathfrak{f}_{v}$ the Lie algebra of $K_{v}$, then $\mathfrak{f}_{v}=\{u \in \mathfrak{f} \mid[u, v]=0\}=$ $\mathfrak{f}_{0} \oplus \bigoplus_{\alpha \in \Lambda^{+}, \alpha(v)=0}^{\mathfrak{f}_{\alpha}}$. 
2.2. Patterson-Sullivan measures. Let $X=G / K$ be a symmetric space of non-compact type, and $\Gamma$ be a cocompact lattice in G. In [1], Albuquerque generalizes the construction of Patterson-Sullivan to higher rank symmetric spaces. He showed that for each $x \in X$, we can assign a probability measure $\mu(x)$ that is $G$-equivariant and is fully supported on the Furstenberg boundary $\partial_{F}(X)$. Moreover, for $x, y \in X$ and $\theta \in \partial_{F}(X)$, the Radon-Nikodym derivative is given by

$$
\frac{d \mu(x)}{d \mu(y)}(\theta)=e^{h B(x, y, \theta)}
$$

where $h$ is the volume entropy of $X / \Gamma$, and $B(x, y, \theta)$ is the Busemann function on $X$. Recall that, in a non-positively curved space $X$, the Busemann function $B$ is defined by

$$
B(x, y, \theta)=\lim _{t \rightarrow \infty}\left(d_{X}\left(y, \gamma_{\theta}(t)\right)-t\right)
$$

where $\gamma_{\theta}$ is the unique geodesic ray from $x$ to $\theta$. Fixing a basepoint $O$ in $X$, we shorten $B(O, y, \theta)$ to just $B(y, \theta)$. Notice that for fixed $\theta \in \partial_{F}(X)$ the Busemann function is convex on $X$, and by integrating on $\partial_{F}(X)$, we obtain, for any probability measure $v$ that is fully supported on the Furstenberg boundary $\partial_{F} X$, a strictly convex function

$$
x \mapsto \int_{\partial_{F} X} B(x, \theta) d v(\theta)
$$

(See [6, Proposition 3.1] for a proof of this last statement.)

Hence we can define the barycenter $\operatorname{bar}(v)$ of $v$ to be the unique point in $X$ where the function attains its minimum. It is clear that this definition is independent of the choice of basepoint $O$.

2.3. Barycenter method. In this section, we discuss the barycentric straightening introduced by Lafont and Schmidt [16] (based on the barycenter method originally developed by Besson, Courtois, and Gallot [3]). Let $X=G / K$ be a symmetric space of non-compact type, and $\Gamma$ be a cocompact lattice in $\mathrm{G}$. We denote by $\Delta_{S}^{k}$ the standard spherical k-simplex in the Euclidean space, that is

$$
\Delta_{s}^{k}=\left\{\left(a_{1}, \ldots, a_{k+1}\right) \mid a_{i} \geq 0, \sum_{i=1}^{k+1} a_{i}^{2}=1\right\} \subseteq \mathbb{R}^{k+1},
$$

with the induced Riemannian metric from $\mathbb{R}^{k+1}$, and with ordered vertices $\left(e_{1}, \ldots, e_{k+1}\right)$. Given any singular k-simplex $f: \Delta_{s}^{k} \rightarrow X$, with ordered vertices $V=\left(x_{1}, \ldots, x_{k+1}\right)=\left(f\left(e_{1}\right), \ldots, f\left(e_{k+1}\right)\right)$, we define the k-straightened simplex

$$
\begin{gathered}
\mathrm{st}_{k}(f): \Delta_{s}^{k} \rightarrow X \\
s t_{k}(f)\left(a_{1}, \ldots, a_{k+1}\right):=\operatorname{bar}\left(\sum_{i=1}^{k+1} a_{i}^{2} \mu\left(x_{i}\right)\right)
\end{gathered}
$$

where $\mu\left(x_{i}\right)$ is the Patterson-Sullivan measure at $x_{i}$. We notice that $s t_{k}(f)$ is determined by the (ordered) vertex set $V$, and we denote $s t_{k}(f)(\delta)$ by $s t_{V}(\delta)$, for $\delta \in \Delta_{s}^{k}$. 
Observe that the map st $k(f)$ is $C^{1}$, since one can view this map as the restriction of the $C^{1}$-map st ${ }_{n}(f)$ to a k-dimensional subspace (see e.g. [16, Property (3)]). For any $\delta=\sum_{i=1}^{k+1} a_{i} e_{i} \in \Delta_{s}^{k}, \mathrm{st}_{k}(f)(\delta)$ is defined to be the unique point where the function

$$
x \mapsto \int_{\partial_{F} X} B(x, \theta) d\left(\sum_{i=1}^{k+1} a_{i}^{2} \mu\left(x_{i}\right)\right)(\theta)
$$

is minimized. Hence, by differentiating at that point, we get the 1 -form equation

$$
\int_{\partial_{F} X} d B_{\left(s t_{V}(\delta), \theta\right)}(\cdot) d\left(\sum_{i=1}^{k+1} a_{i}^{2} \mu\left(x_{i}\right)\right)(\theta) \equiv 0
$$

which holds identically on the tangent space $T_{s t_{V}(\delta)} X$. Differentiating in a direction $u \in T_{\delta}\left(\Delta_{s}^{k}\right)$ in the source, one obtains the 2-form equation

$$
\sum_{i=1}^{k+1} 2 a_{i}\left\langle u, e_{i}\right\rangle_{\delta} \int_{\partial_{F} X} d B_{\left(s t_{V}(\delta), \theta\right)}(v) d\left(\mu\left(x_{i}\right)\right)(\theta)+\int_{\partial_{F} X} D d B_{\left(s t_{V}(\delta), \theta\right)}\left(D_{\delta}\left(s t_{V}\right)(u), v\right) d\left(\sum_{i=1}^{k+1} a_{i}^{2} \mu\left(x_{i}\right)\right)(\theta) \equiv 0
$$

which holds for every $u \in T_{\delta}\left(\Delta_{s}^{k}\right)$ and $v \in T_{s t_{V}(\delta)}(X)$. Now we define two semi-positive definite quadratic forms $Q_{1}$ and $Q_{2}$ on $T_{s t_{V}(\delta)}(X)$ :

$$
\begin{gathered}
Q_{1}(v, v)=\int_{\partial_{F} X} d B_{\left(s t_{V}(\delta), \theta\right)}^{2}(v) d\left(\sum_{i=1}^{k+1} a_{i}^{2} \mu\left(x_{i}\right)\right)(\theta) \\
Q_{2}(v, v)=\int_{\partial_{F} X} D d B_{\left(s t_{V}(\delta), \theta\right)}(v, v) d\left(\sum_{i=1}^{k+1} a_{i}^{2} \mu\left(x_{i}\right)\right)(\theta)
\end{gathered}
$$

In fact, $Q_{2}$ is positive definite since $\sum_{i=1}^{k+1} a_{i}^{2} \mu\left(x_{i}\right)$ is fully supported on $\partial_{F} X$ (see [6, Section 4]). From Equation (2.1), we obtain, for $u \in T_{\delta}\left(\Delta_{s}^{k}\right)$ a unit vector and $v \in T_{s t_{V}(\delta)}(X)$ arbitrary, the following

$$
\begin{aligned}
\left|Q_{2}\left(D_{\delta}\left(s t_{V}\right)(u), v\right)\right| & =\left|-\sum_{i=1}^{k+1} 2 a_{i}\left\langle u, e_{i}\right\rangle_{\delta} \int_{\partial_{F} X} d B_{\left(s t_{V}(\delta), \theta\right)}(v) d\left(\mu\left(x_{i}\right)\right)(\theta)\right| \\
& \leq\left(\sum_{i=1}^{k+1}\left\langle u, e_{i}\right\rangle_{\delta}^{2}\right)^{1 / 2}\left(\sum_{i=1}^{k+1} 4 a_{i}^{2}\left(\int_{\partial_{F} X} d B_{\left(s t_{V}(\delta), \theta\right)}(v) d\left(\mu\left(x_{i}\right)\right)(\theta)\right)^{2}\right)^{1 / 2} \\
& \leq 2\left(\sum_{i=1}^{k+1} a_{i}^{2} \int_{\partial_{F} X} d B_{\left(s t_{V}(\delta), \theta\right)}^{2}(v) d\left(\mu\left(x_{i}\right)\right)(\theta) \int_{\partial_{F} X} 1 d\left(\mu\left(x_{i}\right)\right)\right)^{1 / 2} \\
& =2 Q_{1}(v, v)^{1 / 2}
\end{aligned}
$$

via two applications of the Cauchy-Schwartz inequality.

We restrict these two quadratic forms to the subspace $S=\operatorname{Im}\left(D_{\delta}\left(s t_{V}\right)\right) \subseteq T_{s t_{V}(\delta)}(X)$, and denote the corresponding $k$-dimensional endomorphisms by $H_{\delta}$ and $K_{\delta}$, that is

$$
Q_{1}(v, v)=\left\langle H_{\delta}(v), v\right\rangle_{s t_{V}(\delta)}
$$




$$
Q_{2}(v, v)=\left\langle K_{\delta}(v), v\right\rangle_{s t_{V}(\delta)}
$$

for all $v \in S$.

For points $\delta \in \Delta_{s}^{k}$ where $s t_{V}$ is nondegenerate, we now pick orthonormal bases $\left\{u_{1}, \ldots, u_{k}\right\}$ on $T_{\delta}\left(\Delta_{s}^{k}\right)$, and $\left\{v_{1}, \ldots, v_{k}\right\}$ on $S \subseteq T_{s t_{V}(\delta)}(X)$. We choose these so that $\left\{v_{i}\right\}_{i=1}^{k}$ are eigenvectors of $H_{\delta}$, and $\left\{u_{1}, \ldots, u_{k}\right\}$ is the resulting basis obtained by applying the orthonormalization process to the collection of pullback vectors $\left\{\left(K_{\delta} \circ D_{\delta}\left(s t_{V}\right)\right)^{-1}\left(v_{i}\right)\right\}_{i=1}^{k}$. So we obtain

$$
\begin{aligned}
\operatorname{det}\left(\left.Q_{2}\right|_{S}\right) \cdot\left|J a c_{\delta}\left(s t_{V}\right)\right| & =\left|\operatorname{det}\left(K_{\delta}\right) \cdot J a c_{\delta}\left(s t_{V}\right)\right| \\
& =\left|\operatorname{det}\left(\left\langle K_{\delta} \circ D_{\delta}\left(s t_{V}\right)\left(u_{i}\right), v_{j}\right\rangle\right)\right|
\end{aligned}
$$

By the choice of bases, the matrix $\left(\left\langle K_{\delta} \circ D_{\delta}\left(s t_{V}\right)\left(u_{i}\right), v_{j}\right\rangle\right)$ is upper triangular, so we have

$$
\begin{aligned}
\left|\operatorname{det}\left(\left\langle K_{\delta} \circ D_{\delta}\left(s t_{V}\right)\left(u_{i}\right), v_{j}\right\rangle\right)\right| & =\left|\prod_{i=1}^{k}\left\langle K_{\delta} \circ D_{\delta}\left(s t_{V}\right)\left(u_{i}\right), v_{i}\right\rangle\right| \\
& \leq \prod_{i=1}^{k} 2\left\langle H_{\delta}\left(v_{i}\right), v_{i}\right\rangle^{1 / 2} \\
& =2^{k} \operatorname{det}\left(H_{\delta}\right)^{1 / 2}=2^{k} \operatorname{det}\left(\left.Q_{1}\right|_{S}\right)^{1 / 2}
\end{aligned}
$$

where the middle inequality is obtained via Equation (2.2). Hence we get the inequality

$$
\left|J a c_{\delta}\left(s t_{V}\right)\right| \leq 2^{k} \cdot \frac{\operatorname{det}\left(\left.Q_{1}\right|_{S}\right)^{1 / 2}}{\operatorname{det}\left(\left.Q_{2}\right|_{S}\right)}
$$

We summarize the above discussion into the following proposition.

Proposition 2.3. Let $Q_{1}, Q_{2}$ be the two positive semidefinite quadratic forms defined as above (note $Q_{2}$ is actually positive definite). Assume there exists a constant $C$ that only depends on $X$, with the property that

$$
\frac{\operatorname{det}\left(\left.Q_{1}\right|_{S}\right)^{1 / 2}}{\operatorname{det}\left(\left.Q_{2}\right|_{S}\right)} \leq C
$$

for any $k$-dimensional subspace $S \subseteq T_{s t_{V}(\delta)} X$. Then the quantity $\left|\operatorname{Jac}\left(s t_{V}\right)(\delta)\right|$ is universally boundedindependent of the choice of $(k+1)$-tuple of points $V \subset X$, and of the point $\delta \in \Delta_{s}^{k}$.

\section{JACobian Estimate}

Let $X=G / K$ be an irreducible symmetric space of non-compact type. We fix an arbitrary point $x \in X$ and identify $T_{X} X$ with $\mathfrak{p}$. Let $\mu$ be a probability measure that is fully supported on the Furstenberg boundary $\partial_{F} X$. Using the same notation as in Section 2.3, we define a semi-positive definite quadratic form $Q_{1}$ and a positive definite quadratic form $Q_{2}$ on $T_{x} X$

$$
\begin{gathered}
Q_{1}(v, v)=\int_{\partial_{F} X} d B_{(x, \theta)}^{2}(v) d \mu(\theta) \\
Q_{2}(v, v)=\int_{\partial_{F} X} D d B_{(x, \theta)}(v, v) d \mu(\theta)
\end{gathered}
$$


for $v \in T_{x}(X)$. We will follow the techniques of Connell and Farb [6], [7], and show the following theorem.

Theorem 3.1. Let $X$ be an irreducible symmetric space of non-compact type excluding $\mathrm{SL}(3, \mathbb{R}) / \mathrm{SO}(3)$ and $\mathrm{SL}(4, \mathbb{R}) / \mathrm{SO}(4)$, and let $r=\operatorname{rank}(X) \geq 2$. If $n=\operatorname{dim}(X)$, then there exists a constant $C$ that only depends on $X$, such that

$$
\frac{\operatorname{det}\left(\left.Q_{1}\right|_{S}\right)^{1 / 2}}{\operatorname{det}\left(\left.Q_{2}\right|_{S}\right)} \leq C
$$

for any subspace $S \subseteq T_{x} X$ with $n-r+2 \leq \operatorname{dim}(S) \leq n$.

In view of Proposition 2.3 , this implies that the barycentrically straightened simplices of dimension $\geq$ $n-r+2$ have uniformly controlled Jacobians. The reader whose primary interest is bounded cohomology, and who is willing to take Theorem 3.1 on faith, can skip ahead to Section 5 for the proof of the Main Theorem.

The rest of this Section will be devoted to the proof of Theorem 3.1. In Section 3.1, we explain some simplifications of the quadratic forms, allowing us to give geometric interpretations for the quantities involved in Theorem 3.1. In Section 3.2, we formulate the "weak eigenvalue matching" Theorem 3.3 (which will be established in Section (4). Finally, in Section 3.3, we will deduce Theorem 3.1 from Theorem 3.3.

3.1. Simplifying the quadratic forms. Following [6, Section 4.3], we fix a flat $\mathcal{F}$ going through $x$, and denote the tangent space by $\mathfrak{a}$, so $\operatorname{dim}(\mathfrak{a})=r$ is the rank of $X$. By abuse of notation, we identify a with $\mathcal{F}$. Choose an orthonormal basis $\left\{e_{i}\right\}$ on $T_{x} X$ such that $\left\{e_{1}, \ldots, e_{r}\right\}$ spans $\mathcal{F}$, and assume $e_{1}$ is regular so that $e_{1}(\infty) \in \partial_{F} X$. Then $Q_{1}, Q_{2}$ can be expressed in the following matrix forms.

$$
\begin{gathered}
Q_{1}=\int_{\partial_{F} X} O_{\theta}\left(\begin{array}{cc}
1 & 0 \\
0 & 0^{(n-1)}
\end{array}\right) O_{\theta}^{*} d \mu(\theta) \\
Q_{2}=\int_{\partial_{F} X} O_{\theta}\left(\begin{array}{cc}
0^{(r)} & 0 \\
0 & D_{\lambda}^{(n-r)}
\end{array}\right) O_{\theta}^{*} d \mu(\theta)
\end{gathered}
$$

where $D_{\lambda}=\operatorname{diag}\left(\lambda_{1}, \ldots, \lambda_{(n-r)}\right)$, and $O_{\theta}$ is the orthogonal matrix corresponding to the unique element in $K$ that sends $e_{1}$ to $v_{(x, \theta)}$, the direction at $x$ pointing towards $\theta$. Moreover, there exists a constant $c>0$ that only depends on $X$, so that $\lambda_{i} \geq c$ for $1 \leq i \leq n-r$. For more details, we refer the readers to the original [6].

Denote by $\bar{Q}_{2}$ the quadratic form given by

$$
\bar{Q}_{2}=\int_{\partial_{F} X} O_{\theta}\left(\begin{array}{cc}
0^{(r)} & 0 \\
0 & I^{(n-r)}
\end{array}\right) O_{\theta}^{*} d \mu(\theta)
$$

Then the difference $Q_{2}-c \bar{Q}_{2}$ is positive semi-definite, hence $\operatorname{det}\left(\left.Q_{2}\right|_{S}\right) \geq \operatorname{det}\left(\left.c \bar{Q}_{2}\right|_{S}\right)$. So in order to show Theorem 3.1, it suffices to assume $Q_{2}$ has the matrix form

$$
\int_{\partial_{F} X} O_{\theta}\left(\begin{array}{cc}
0^{(r)} & 0 \\
0 & I^{(n-r)}
\end{array}\right) O_{\theta}^{*} d \mu(\theta)
$$


Given any $v \in T_{x} X$, we have the following geometric estimates on the value of the quadratic form

$$
\begin{aligned}
Q_{1}(v, v) & =\int_{\partial_{F} X} v^{t} O_{\theta}\left(\begin{array}{cc}
1 & 0 \\
0 & 0^{(n-1)}
\end{array}\right) O_{\theta}^{*} v d \mu(\theta) \\
& =\int_{\partial_{F} X}\left\langle O_{\theta}^{*} v, e_{1}\right\rangle^{2} d \mu(\theta) \\
& \leq \int_{\partial_{F} X} \sum_{i=1}^{r}\left\langle O_{\theta}^{*} v, e_{i}\right\rangle^{2} d \mu(\theta) \\
& =\int_{\partial_{F} X} \sin ^{2}\left(\angle\left(O_{\theta}^{*} v, \mathcal{F}^{\perp}\right)\right) d \mu(\theta)
\end{aligned}
$$

Roughly speaking, $Q_{1}(v, v)$ is bounded above by the weighted average of the time the $K$-orbit spends away from $\mathcal{F}^{\perp}$. Similarly we can estimate

$$
\begin{aligned}
Q_{2}(v, v) & =\int_{\partial_{F} X} v^{t} O_{\theta}\left(\begin{array}{cc}
0^{(r)} & 0 \\
0 & I^{(n-r)}
\end{array}\right) O_{\theta}^{*} v d \mu(\theta) \\
& =\int_{\partial_{F} X} \sum_{i=r+1}^{n}\left\langle O_{\theta}^{*} v, e_{i}\right\rangle^{2} d \mu(\theta) \\
& =\int_{\partial_{F} X} \sin ^{2}\left(\angle\left(O_{\theta}^{*} v, \mathcal{F}\right)\right) d \mu(\theta)
\end{aligned}
$$

So again, $Q_{2}(v, v)$ roughly measures the weighted average of the time the $K$-orbit spends away from $\mathcal{F}$.

3.2. Eigenvalue matching. In their original paper, Connell and Farb showed an eigenvalue matching theorem [6. Theorem 4.4], in order to get the Jacobian estimate in top dimension. For the small eigenvalues of $Q_{2}$ (there are at most $r$ of them), they want to find twice as many comparatively small eigenvalues of $Q_{1}$. Then by taking the product of those eigenvalues, they obtain a uniform upper bound on the ratio of determinants $\operatorname{det}\left(Q_{1}\right)^{1 / 2} / \operatorname{det}\left(Q_{2}\right)$, which yields an upper bound on the Jacobian. However, as was pointed out by Inkang Kim and Sungwoon Kim, there was a mistake in the proof. Connell and Farb fixed the gap by showing a weak eigenvalue matching theorem [7, Theorem 0.1], which was sufficient to imply the Jacobian inequality.

We generalize this method and show that in fact we can find $(r-2)$ additional small eigenvalues of $Q_{1}$ that are bounded by a universal constant times the smallest eigenvalue of $Q_{2}$. This allows for the Jacobian inequality to be maintained when we pass down to a subspace of codimension at most $(r-2)$. We now state our version of the weak eigenvalue matching theorem.

Definition 3.2. We call a set of unit vectors $\left\{w_{1}, \ldots, w_{k}\right\}$ a $\delta$-orthonormal $k$-frame if $\left\langle w_{i}, w_{j}\right\rangle\langle\delta$ for all $1 \leq i<j \leq k$.

Theorem 3.3. (Weak eigenvalue matching.) Let $X$ be an irreducible symmetric space of non-compact type, with $r=\operatorname{rank}(X) \geq 2$, excluding $\mathrm{SL}(3, \mathbb{R}) / \mathrm{SO}(3)$ and $\mathrm{SL}(4, \mathbb{R}) / \mathrm{SO}(4)$. There exist constants $C^{\prime}, C$, $\delta$ that 
only depend on $X$ so that the following holds. Given any $\epsilon<\delta$, and any orthonormal $k$-frame $\left\{v_{1}, \ldots, v_{k}\right\}$ in $T_{x} X$ with $k \leq r$, whose span $V$ satisfies $\angle(V, \mathcal{F}) \leq \epsilon$, then there is $a\left(C^{\prime} \epsilon\right)$-orthonormal $(2 k+r-2)$-frame given by vectors $\left\{v_{1}^{\prime}, v_{1}^{\prime \prime}, \ldots, v_{1}^{(r)}, v_{2}^{\prime}, v_{2}^{\prime \prime}, \ldots, v_{k}^{\prime}, v_{k}^{\prime \prime}\right\}$, such that for $i=1, \ldots, k$, and $j=1, \ldots, r$, we have the following inequalities:

$$
\begin{gathered}
\angle\left(h v_{i}^{\prime}, \mathcal{F}^{\perp}\right) \leq C \angle\left(h v_{i}, \mathcal{F}\right) \\
\angle\left(h v_{i}^{\prime \prime}, \mathcal{F}^{\perp}\right) \leq C \angle\left(h v_{i}, \mathcal{F}\right) \\
\angle\left(h v_{1}^{(j)}, \mathcal{F}^{\perp}\right) \leq C \angle\left(h v_{1}, \mathcal{F}\right)
\end{gathered}
$$

for all $h \in K$, where $h v$ is the linear action of $h \in K$ on $v \in T_{x} X \simeq \mathfrak{p}$.

The proof of Theorem 3.3 will be delayed to Section 4

3.3. Proof of Theorem 3.1. In this section, we will prove Theorem 3.1 using Theorem 3.3 Before starting the proof, we will need the following three elementary results from linear algebra.

Lemma 3.4. Let $Q$ be a positive definite quadratic form on some Euclidean space $V$ of dimension $n$, with eigenvalues $\lambda_{1} \leq \lambda_{2} \leq \ldots \leq \lambda_{n}$. Let $W \subseteq V$ be a subspace of codimension l, and let $\mu_{1} \leq \mu_{2} \leq \ldots \leq \mu_{n-l}$ be the eigenvalues of $Q$ restricted to $W$. Then $\lambda_{i} \leq \mu_{i} \leq \lambda_{i+l}$ holds for $i=1, \ldots, n-l$.

Proof. We argue by contradiction. Assume $\mu_{i}>\lambda_{i+l}$ for some $i$. Take the subspace $W_{0} \subseteq W$ spanned by the eigenvectors corresponding to $\mu_{i}, \mu_{i+1}, \ldots, \mu_{n-l}$; clearly $\operatorname{dim}\left(W_{0}\right)=n-l-i+1$. So for any nonzero vectors $v \in W_{0}$, we have $Q(v, v) \geq \mu_{i}\|v\|^{2}>\lambda_{i+l}\|v\|^{2}$. However, if we denote $V_{0} \subseteq V$ the $(i+l)$-dimensional subspace spanned by the eigenvectors corresponding to $\lambda_{1}, \ldots, \lambda_{i+l}$, we have $Q(v, v) \leq \lambda_{i+l}\|v\|^{2}$ for any $v \in V_{0}$. But $\operatorname{dim}\left(W_{0} \cap V_{0}\right) \geq \operatorname{dim}\left(W_{0}\right)+\operatorname{dim}\left(V_{0}\right)-\operatorname{dim}(V)=1$ implies $W_{0} \cap V_{0}$ is nontrivial, so we obtain a contradiction. This establishes $\mu_{i} \leq \lambda_{i+l}$. A similar argument shows $\lambda_{i} \leq \mu_{i}$.

Lemma 3.5. Let $Q$ be a positive definite quadratic form on some Euclidean space $V$ of dimension $n$, with eigenvalues $\lambda_{1} \leq \lambda_{2} \leq \cdots \leq \lambda_{n}$. If $\left\{v_{1}, \ldots, v_{n}\right\}$ is any orthonormal frame of $V$, ordered so that $Q\left(v_{1}, v_{1}\right) \leq$ $Q\left(v_{2}, v_{2}\right) \leq \cdots \leq Q\left(v_{n}, v_{n}\right)$, then $Q\left(v_{i}, v_{i}\right) \geq \lambda_{i} / n$ for $i=1, \ldots, n$.

Proof. We show this by induction on the dimension of $V$. The statement is clear when $n=1$, so let us now assume we have the statement for $\operatorname{dim}(V)=n-1$. Now if $\operatorname{dim}(V)=n$, we restrict the quadratic form $Q$ to the $(n-1)$-dimensional subspace $W$ spanned by $v_{1}, \ldots, v_{n-1}$, and denote the eigenvalues of $\left.Q\right|_{W}$ by $\mu_{1} \leq \mu_{2} \leq \ldots \leq \mu_{n-1}$. By the induction hypothesis and Lemma 3.4, we obtain

$$
Q\left(v_{i}, v_{i}\right) \geq \frac{\mu_{i}}{n-1} \geq \frac{\lambda_{i}}{n-1} \geq \frac{\lambda_{i}}{n}
$$

for $1 \leq i \leq n-1$. Finally, for the last vector, we have

$$
Q\left(v_{n}, v_{n}\right) \geq \frac{Q\left(v_{1}, v_{1}\right)+\ldots+Q\left(v_{n}, v_{n}\right)}{n}=\frac{\operatorname{tr}(Q)}{n}=\frac{\lambda_{1}+\ldots+\lambda_{n}}{n} \geq \frac{\lambda_{n}}{n}
$$

This completes the proof of the lemma. 
Lemma 3.6. Let $Q$ be a positive definite quadratic form on some Euclidean space $V$ of dimension $n$. If $\left\{v_{1}, \ldots, v_{k}\right\}$ is any $\tau$-orthonormal $k$-frame for $\tau$ sufficiently small (only depends on $n$ ), ordered so that $Q\left(v_{1}, v_{1}\right) \leq \ldots \leq Q\left(v_{k}, v_{k}\right)$, then there is an orthonormal $k$-frame $\left\{u_{1}, \ldots, u_{k}\right\}$ such that $Q\left(u_{i}, u_{i}\right) \leq 2 Q\left(v_{i}, v_{i}\right)$.

Proof. We do the Gram-Schmidt process on $\left\{v_{1}, \ldots, v_{k}\right\}$ and obtain an orthonormal $k$-frame $\left\{u_{1}, \ldots, u_{k}\right\}$. Notice $\left\{v_{1}, \ldots, v_{k}\right\}$ is $\tau$-orthonormal, so we have $u_{i}=v_{i}+O(\tau) v_{1}+\ldots+O(\tau) v_{i}$, where by $O(\tau)$ we denote a number that has universal bounded (only depends on $n$ ) ratio with $\tau$. This implies

$$
Q\left(u_{i}, u_{i}\right)=Q\left(v_{i}, v_{i}\right)+O(\tau) \sum_{1 \leq s \leq t \leq i} Q\left(v_{s}, v_{t}\right)
$$

Since $\left|Q\left(v_{s}, v_{t}\right)\right| \leq \sqrt{Q\left(v_{s}, v_{s}\right) Q\left(v_{t}, v_{t}\right)} \leq Q\left(v_{i}, v_{i}\right)$, we obtain

$$
Q\left(u_{i}, u_{i}\right) \leq Q\left(v_{i}, v_{i}\right)+O(\tau) Q\left(v_{i}, v_{i}\right) \leq 2 Q\left(v_{i}, v_{i}\right)
$$

for $\tau$ sufficiently small. This completes the proof of the lemma.

We are now ready to establish Theorem 3.1

Proof. As was shown in [6, Section 4.4], for any fixed $\epsilon_{0} \leq 1 /(r+1)$, there are at most $r$ eigenvalues of $Q_{2}$ that are smaller than $\epsilon_{0}$ (we will choose $\epsilon_{0}$ in the course of the proof). By Lemma 3.4 the same is true for $\left.Q_{2}\right|_{S}$. We arrange these small eigenvalues in the order $L_{1} \leq L_{2} \leq \ldots \leq L_{k}$, where $k \leq r$. Observe that, if no such eigenvalue exists, then by Lemma 3.4, $\operatorname{det}\left(\left.Q_{2}\right|_{S}\right)$ is uniformly bounded below, and the theorem holds (since the eigenvalues of $\left.Q_{1}\right|_{S}$ are all $\leq 1$ ). So we will henceforth assume $k \geq 1$. We denote the corresponding unit eigenvectors by $v_{1}, \ldots, v_{k}$ (so that $v_{i}$ has eigenvalue $L_{i}$ ). Although $V=\operatorname{span}\left\{v_{1}, \ldots v_{k}\right\}$ might not have small angle with $\mathcal{F}$, it is shown in [7, Section 3] that there is a $k_{0} \in K$ so that $\angle\left(k_{0} v_{i}, \mathcal{F}\right) \leq$ $2 \epsilon_{0}^{1 / 4}$ for each $i$.

Let $\epsilon$ be a constant small enough so that $\epsilon<\delta$, where $\delta$ is from Theorem 3.3. and also $\tau:=C^{\prime} \epsilon$ satisfies the condition of Lemma 3.6 (where $C^{\prime}$ is obtained from Theorem 3.3). Hence the choice of $\epsilon$ only depends on $X$. We now make a choice of $\epsilon_{0}$ such that $2 \epsilon_{0}^{1 / 4}<\epsilon$, and hence $\angle\left(k_{0} V, \mathcal{F}\right)<\epsilon$. (Note again the choice of $\epsilon_{0}$ only depends on $X$.)

Apply Theorem 3.3 to the frame $\left\{k_{0} v_{1}, \ldots, k_{0} v_{k}\right\}$, and translate the obtained $\left(C^{\prime} \epsilon\right)$-orthonormal frame by $k_{0}^{-1}$. This gives us a $\left(C^{\prime} \epsilon\right)$-orthonormal $(2 k+r-2)$-frame $\left\{v_{1}^{\prime}, v_{1}^{\prime \prime}, \ldots, v_{1}^{(r)}, v_{2}^{\prime}, v_{2}^{\prime \prime}, \ldots, v_{k}^{\prime}, v_{k}^{\prime \prime}\right\}$, such that for $i=1, \ldots, k$, and $j=1, \ldots r$, we have

$$
\begin{gathered}
\angle\left(h v_{i}^{\prime}, \mathcal{F}^{\perp}\right) \leq C \angle\left(h v_{i}, \mathcal{F}\right) \\
\angle\left(h v_{i}^{\prime \prime}, \mathcal{F}^{\perp}\right) \leq C \angle\left(h v_{i}, \mathcal{F}\right) \\
\angle\left(h v_{1}^{(j)}, \mathcal{F}^{\perp}\right) \leq C \angle\left(h v_{1}, \mathcal{F}\right)
\end{gathered}
$$

for all $h \in K$ (note that we have absorbed the $k_{0}$-translation into the element $h$ ).

We notice $\angle\left(h v_{i}^{\prime}, \mathcal{F}^{\perp}\right) \leq C \angle\left(h v_{i}, \mathcal{F}\right)$ implies $\sin ^{2}\left(\angle\left(h v_{i}^{\prime}, \mathcal{F}^{\perp}\right)\right) \leq C_{0} \sin ^{2}\left(\angle\left(h v_{i}, \mathcal{F}\right)\right)$ for some $C_{0}$ depending on $C$. For convenience, we still use $C$ for this new constant. Hence, we obtain

$$
Q_{1}\left(v_{i}^{\prime}, v_{i}^{\prime}\right) \leq \int_{\partial_{F} X} \sin ^{2}\left(\angle\left(O_{\theta}^{*} v_{i}^{\prime}, \mathcal{F}^{\perp}\right)\right) d \mu(\theta)
$$




$$
\leq C \int_{\partial_{F} X} \sin ^{2}\left(\angle\left(O_{\theta}^{*} v_{i}, \mathcal{F}\right)\right) d \mu(\theta)=C Q_{2}\left(v_{i}, v_{i}\right)=C L_{i}
$$

An identical estimate gives us $Q_{1}\left(v_{i}^{\prime \prime}, v_{i}^{\prime \prime}\right) \leq C L_{i}$, and $Q_{1}\left(v_{1}^{(j)}, v_{1}^{(j)}\right) \leq C L_{1}$.

We rearrange the $\left(C^{\prime} \epsilon\right)$-orthonormal $(2 k+r-2)$-frame as $\left\{u_{1}^{\prime}, u_{1}^{\prime \prime}, \ldots, u_{1}^{(r)}, u_{2}^{\prime}, u_{2}^{\prime \prime}, \ldots, u_{k}^{\prime}, u_{k}^{\prime \prime}\right\}$ so that it has increasing order when applying $Q_{1}$. Then the inequalities still hold for this new frame:

$$
\begin{gathered}
Q_{1}\left(u_{i}^{\prime}, u_{i}^{\prime}\right) \leq C L_{i} \\
Q_{1}\left(u_{i}^{\prime \prime}, u_{i}^{\prime \prime}\right) \leq C L_{i} \\
Q_{1}\left(u_{1}^{(j)}, u_{1}^{(j)}\right) \leq C L_{1}
\end{gathered}
$$

Since the choice of $\epsilon$ makes $C^{\prime} \epsilon$ satisfy the condition of Lemma 3.6, we apply the lemma to this $C^{\prime} \epsilon$ orthonormal frame. This gives us an orthonormal $(2 k+r-2)$-frame $\left\{\overline{u_{1}^{\prime}}, \overline{u_{1}^{\prime \prime}}, \ldots, \overline{u_{1}^{(r)}}, \overline{u_{2}^{\prime}}, \overline{u_{2}^{\prime \prime}}, \ldots, \overline{u_{k}^{\prime}}, \overline{u_{k}^{\prime \prime}}\right\}$, such that

$$
\begin{gathered}
Q_{1}\left(\overline{u_{i}^{\prime}}, \overline{u_{i}^{\prime}}\right) \leq 2 Q_{1}\left(u_{i}^{\prime}, u_{i}^{\prime}\right) \leq 2 C L_{i} \\
Q_{1}\left(\overline{u_{i}^{\prime \prime}}, \overline{u_{i}^{\prime \prime}}\right) \leq 2 Q_{1}\left(u_{i}^{\prime \prime}, u_{i}^{\prime \prime}\right) \leq 2 C L_{i} \\
Q_{1}\left(\overline{u_{1}^{(j)}}, \overline{u_{1}^{(j)}}\right) \leq 2 Q_{1}\left(u_{1}^{(j)}, u_{1}^{(j)}\right) \leq 2 C L_{1}
\end{gathered}
$$

Again, we can rearrange the orthonormal basis to have increasing order when applying $Q_{1}$, and it is easy to check that, for the resulting rearranged orthonormal basis, the same inequalities still hold.

We denote the first $(2 k+r-2)$ eigenvalues of $Q_{1}$ by $\lambda_{1}^{\prime} \leq \lambda_{1}^{\prime \prime} \leq \ldots \leq \lambda_{1}^{(r)} \leq \lambda_{2}^{\prime} \leq \lambda_{2}^{\prime \prime} \leq \ldots \leq \lambda_{k}^{\prime} \leq \lambda_{k}^{\prime \prime}$, and the first $2 k$ eigenvalues of $\left.Q_{1}\right|_{S}$ by $\mu_{1}^{\prime} \leq \mu_{1}^{\prime \prime} \leq \ldots \leq \mu_{k}^{\prime} \leq \mu_{k}^{\prime \prime}$. Applying Lemma 3.5, we have

$$
\begin{gathered}
\lambda_{i}^{\prime} \leq n Q_{1}\left(\overline{u_{i}^{\prime}}, \overline{u_{i}^{\prime}}\right) \leq 2 n C L_{i} \\
\lambda_{i}^{\prime \prime} \leq n Q_{1}\left(\overline{u_{i}^{\prime \prime}}, \overline{u_{i}^{\prime \prime}}\right) \leq 2 n C L_{i} \\
\lambda_{1}^{(j)} \leq n Q_{1}\left(\overline{u_{1}^{(j)}}, \overline{u_{1}^{(j)}}\right) \leq 2 n C L_{1}
\end{gathered}
$$

for $1 \leq i \leq k$ and $1 \leq j \leq l$.

Notice $\operatorname{dim}(S) \geq n-r+2$. We apply Lemma 3.4 and obtain

$$
\begin{gathered}
\mu_{1}^{\prime} \leq \lambda_{1}^{(r-1)} \leq 2 n C L_{1} \\
\mu_{1}^{\prime \prime} \leq \lambda_{1}^{(r)} \leq 2 n C L_{1} \\
\mu_{i}^{\prime} \leq \lambda_{i}^{\prime} \leq 2 n C L_{i} \\
\mu_{i}^{\prime \prime} \leq \lambda_{i}^{\prime \prime} \leq 2 n C L_{i}
\end{gathered}
$$

for $2 \leq i \leq k$. The eigenvalues of $\left.Q_{1}\right|_{S}$ are bounded above by 1 , and $L_{1}, \ldots, L_{k}$ are the only eigenvalues of $\left.Q_{2}\right|_{S}$ that are below $\epsilon_{0}$ (and recall the choice of $\epsilon_{0}$ only depends on $X$ ). Therefore,

$$
\begin{aligned}
\operatorname{det}\left(\left.Q_{1}\right|_{S}\right) \leq \prod_{i=1}^{k} \mu_{i}^{\prime} \mu_{i}^{\prime \prime} & \leq \prod_{i=1}^{k}\left(2 n C L_{i}\right)^{2} \leq(2 n C)^{2 k}\left[\frac{\operatorname{det}\left(\left.Q_{2}\right|_{S}\right)}{\epsilon_{0}^{\operatorname{dim}(S)-k}}\right]^{2} \\
& \leq \bar{C} \operatorname{det}\left(\left.Q_{2}\right|_{S}\right)^{2}
\end{aligned}
$$


where $\bar{C}$ only depends on $X$. This completes the proof of Theorem 3.1 .

\section{Reduction to the Combinatorial Problem}

In this section, we will prove the "weak eigenvalue matching" Theorem 3.3, which was introduced in Section 3.2. The approach is to follow [7], and reduce the theorem to a combinatorial problem. Then we apply Hall's Marriage theorem to solve it.

4.1. Hall's Marriage Theorem. We introduce the classic Hall's Marriage Theorem, and later on we will apply a slightly stronger version (Corollary 4.3 below) in the proof of Lemma 4.5 .

Theorem 4.1 (Hall's Marriage Theorem). Suppose we have a set of $m$ different species $A=\left\{a_{1}, . ., a_{m}\right\}$, and a set of $n$ different planets $B=\left\{b_{1}, \ldots, b_{n}\right\}$. Let $\phi: A \rightarrow \mathcal{P}(B)$ be a map which sends a species to the set of all suitable planets for its survival. Then we can arrange for each species a different planet to survive if and only if for any subset $A_{0} \subseteq A$, we have the cardinality inequality $\left|\phi\left(A_{0}\right)\right| \geq\left|A_{0}\right|$.

Corollary 4.2. Under the assumption of Theorem 4.1 we can arrange for each species two different planets if and only if for any subset $A_{0} \subseteq A$, we have the cardinality inequality $\left|\phi\left(A_{0}\right)\right| \geq 2\left|A_{0}\right|$.

Proof. Assume there exists such arrangement, the cardinality condition holds obviously. On the other hand, assume we have the cardinality condition, we want to show there is an arrangement. We make an identical copy on each species and form the set $A^{\prime}=\left\{a_{1}^{\prime}, \ldots, a_{m}^{\prime}\right\}$. We apply the Hall's Marriage Theorem to the set $A \cup A^{\prime}$ relative to $B$. Then for each $i$, both species $a_{i}$ and $a_{i}^{\prime}$ have its own planet, and that means there are two planets for the original species $a_{i}$.

To see why the cardinality condition holds, we choose an arbitrary subset $H \cup K^{\prime} \subseteq A \cup A^{\prime}$ where $H \subseteq A$ and $K^{\prime} \subseteq A^{\prime}$. Let $K$ be the corresponding identical copy of $K^{\prime}$ in $A$. We have $\phi\left(H \cup K^{\prime}\right)=\phi(H \cup K) \geq$ $2|H \cup K| \geq|H|+|K|=\left|H \cup K^{\prime}\right|$. This completes the proof.

Corollary 4.3. Suppose we have a set of vectors $V=\left\{v_{1}, \ldots, v_{r}\right\}$, and for each $v_{i}$, the selectable set is denoted by $B_{i} \subseteq B$. If for any subset $V_{0}=\left\{v_{i_{1}}, \ldots, v_{i_{k}}\right\} \subseteq V$, we have $\left|B_{i_{1}} \cup \ldots \cup B_{i_{k}}\right| \geq 2 k+r-2$, then we can pick $(3 r-2)$ distinct element $\left\{b_{1}^{\prime}, \ldots, b_{1}^{(r)}, b_{i}^{\prime}, b_{i}^{\prime \prime}(2 \leq i \leq r)\right\}$ in $B$ such that $b_{1}^{\prime}, \ldots, b_{1}^{(r)} \in B_{1}$ and $b_{i}^{\prime}, b_{i}^{\prime \prime} \in B_{i}$.

Proof. First we choose $V_{0}$ the singleton set that consists of only $v_{1}$. By hypothesis, we have $\left|B_{1}\right| \geq r \geq(r-2)$, hence we are able to choose $(r-2)$ elements $b_{1}^{(3)}, \ldots, b_{1}^{(r)}$ for $v_{1}$. Next we can easily check the cardinality condition and apply Corollary 4.2 to the set $V$ with respect to $B \backslash\left\{b_{1}^{(3)}, \ldots, b_{1}^{(r)}\right\}$ to obtain the pairs $\left\{b_{i}^{\prime}, b_{i}^{\prime \prime}\right\}$ (for each $1 \leq i \leq r$ ). This completes the proof of this corollary.

4.2. Angle inequality. Throughout this section, we will work exclusively with unit vectors in $T_{x} X \simeq \mathfrak{p}$. We embed the point stabilizer $K_{x}$ into $\operatorname{Isom}\left(T_{x} X\right) \simeq O(n)$, and endow it with the induced metric. This gives rise to a norm on $K$, defined by $\|k\|=\max _{v \in T_{x} X} \angle(v, k v), \forall k \in K$. We denote the Lie algebra of $K_{x} \simeq K$ by $\mathfrak{f}$, which has root space decomposition $\mathfrak{f}=\mathfrak{I}_{0} \oplus \bigoplus_{\alpha \in \Lambda^{+}} \mathfrak{f}_{\alpha}$. For each small element $k \in K$, the action on a vector $v$ can be approximated by the Lie algebra action, that is, if $k=\exp (u)$ is small, then 
$\|[u, v]\| \approx\|k v-v\| \sim \angle(v, k v)$, where we write $A \sim B$ if $A / B$ and $B / A$ are both universally bounded. By abuse of notation, we do not distinguish between $\|k\|$ and $\|u\|$ inside a very small neighborhood $\mathcal{U}$ of 0 inside $\mathfrak{f}$. Although $\|\cdot\|$ is not linear on $\mathcal{U}$, it is linear up to a universal constant, that is, $\|t u\| \sim t\|u\|$, for all $u \in \mathcal{U}$ and $t$ such that $t u \in \mathcal{U}$. We now show the following lemmas.

Lemma 4.4. (Compare [7, Lemma 1.1]) Let $X=G / K$ be a rank $r \geq 2$ irreducible symmetric space of non-compact type, and fix a flat $\mathcal{F} \subseteq T_{X} X$ at $x$. Then for any small $\rho>0$, there is a constant $C(\rho)$ with the following property. If $v \in \mathcal{F}$ is arbitrary, and $v^{*} \in \mathcal{F}$ is a maximally singular vector in the $\rho$-neighborhood of $v$ (in the sense that the dimension of $K_{v^{*}}$ is as large as possible), then

$$
\angle\left(h u, \mathcal{F}^{\perp}\right) \leq C \angle(h v, \mathcal{F})
$$

for any $h \in K$, and $u \in\left(K_{v^{*}} \mathcal{F}\right)^{\perp} \simeq \bigoplus_{\alpha \in \Lambda^{+}, \alpha\left(v^{*}\right) \neq 0} \mathfrak{p}_{\alpha}$, where $\Lambda^{+}$is the set of all positive roots. Moreover, we have

$$
\angle\left(h u, \mathcal{F}^{\perp}\right) \leq C \angle\left(h k_{0} v, K_{v^{*}} \mathcal{F}\right)
$$

for any $h \in K, u \in\left(K_{v^{*}} \mathcal{F}\right)^{\perp}$, and $k_{0} \in K_{v^{*}}$.

Proof. We only need to verify the inequality when $\angle(h v, \mathcal{F})$ is small. Notice for any vector $v \in \mathcal{F}$, and any small element $w \in \mathfrak{f}_{\alpha}=(I+\theta) \mathfrak{g}_{\alpha}=(I+\theta)(I-\theta)^{-1} \mathfrak{p}_{\alpha}$, the Lie algebra action (see Proposition 2.2) has norm

$$
\|[w, v]\|=\left\|-\alpha(v) \cdot(I-\theta)(I+\theta)^{-1} w\right\| \sim|\alpha(v)| \cdot\|w\| .
$$

This is due to the fact that $(I+\theta)(I-\theta)^{-1}$ is a linear isomorphism between $\mathfrak{f}_{\alpha}$ and $\mathfrak{p}_{\alpha}$ (see Proposition 2.1), and when restricted to $\mathfrak{f}_{\alpha} \cap \mathcal{U}$, it preserves the norms up to a uniform multiplicative constant.

Infinitesimally speaking, for $h=\exp (w)$, we have that $h v-v=[w, v]$, so the estimate on the Lie algebra action tells us about the infinitesimal growth of $\|h v-v\|$. We also see that, since $[w, v] \in \mathfrak{p}_{\alpha}, h$ moves the vector $v$ in the direction $\mathfrak{p}_{\alpha}$ (which we recall is orthogonal to the flat $\mathcal{F}$, see Proposition 2.1). Now $v^{*}$ is a maximally singular vector in the $\rho$-neighborhood of the unit vector $v$, so once $\rho$ is small enough, if $\alpha$ is any root with $\alpha\left(v^{*}\right) \neq 0$, then $\alpha(v)$ will be uniformly bounded away from zero (depending only on the choice of $\rho)$. This shows that if a root $\alpha$ satisfies $\alpha\left(v^{*}\right) \neq 0$, then $\angle(h v, \mathcal{F}) \sim\|h\|$ for all $h \in \exp \left(\mathfrak{f}_{\alpha} \cap \mathcal{U}\right)$.

Now we move to analyzing the general case $h=\exp (w)$, where $w \in \mathfrak{f}$ is arbitrary. If $\angle(h v, \mathcal{F})$ is small, then it follows that the components of $h v$ on each $\mathfrak{p}_{\alpha}$ must be small. From the discussion above, this implies that the component of $w$ in each $\left.\mathfrak{f}_{\alpha}\right|_{\alpha\left(v^{*}\right) \neq 0}$ is small, i.e. $w$ almost lies in $\mathfrak{f}_{v^{*}}=\mathfrak{f}_{0} \oplus \bigoplus_{\alpha\left(v^{*}\right)=0} \mathfrak{f}_{\alpha}$. Since $h$ almost lies in $K_{v^{*}}$, there exists an element $h_{0} \in K_{v^{*}}$ such that $h_{0}^{-1} h$ is close to the identity. We write $h=h_{0} h_{1}$, where $h_{1}=\exp \left(w_{1}\right) \in \exp \left(\mathfrak{f}_{v^{*}}^{\perp}\right)=\exp \left(\bigoplus_{\alpha\left(v^{*}\right) \neq 0} \mathfrak{f}_{\alpha}\right)$, and observe that the analysis in the previous paragraph applies to the element $h_{1}$. Now observe that, infinitesimally, $h_{1} v-v=\left[u_{1}, v\right] \in \bigoplus_{\alpha\left(v^{*}\right) \neq 0} \mathfrak{p}_{\alpha}$, so $h_{1}$ moves $v$ in a direction lying in $\bigoplus_{\alpha\left(v^{*}\right) \neq 0} \mathfrak{p}_{\alpha}$. On the other hand, infinitesimally, $K_{v^{*}}$ moves the entire flat $\mathcal{F}$ in the directions $\bigoplus_{\alpha\left(v^{*}\right)=0} \mathfrak{p}_{\alpha}$ (corresponding to the action of its Lie algebra $\mathfrak{f}_{v_{*}}$ ). But these two directions are orthogonal, which means that $h_{1} v$ leaves not just $\mathcal{F}$ orthogonally, but actually leaves orthogonally to the entire orbit $K_{v^{*}} \mathcal{F}$. This allows us to estimate

$$
\angle(h v, \mathcal{F})=\angle\left(h_{1} v, h_{0}^{-1} \mathcal{F}\right) \geq \angle\left(h_{1} v, K_{v^{*}} \mathcal{F}\right) \sim\left\|h_{1}\right\|,
$$


where at the last step, we use that $h_{1}$ moves $v$ orthogonally off the $K_{v^{*}}$ orbit of $\mathcal{F}$. On the other hand, we are assuming that the vector $u$ lies in $\left(K_{v^{*}} \mathcal{F}\right)^{\perp}$, hence also in $h_{0}^{-1} \mathcal{F}^{\perp}$. So we have the sequence of inequalities

$$
\angle\left(h u, \mathcal{F}^{\perp}\right)=\angle\left(h_{1} u, h_{0}^{-1} \mathcal{F}^{\perp}\right) \leq \angle\left(h_{1} u, u\right) \leq\left\|h_{1}\right\| .
$$

Combining equations (4.2) and (4.3) gives us the first inequality.

Similarly, $\angle\left(h k_{0} v, K_{v^{*}} \mathcal{F}\right)$ being small also implies that the component of $h$ on each $\left.\mathfrak{f}_{\alpha}\right|_{\alpha\left(v^{*}\right) \neq 0}$ is small. So by writing $h=h_{0} h_{1}$ in the same manner, we get $\angle\left(h k_{0} v, K_{v^{*}} \mathcal{F}\right)=\angle\left(h_{1} k_{0} v, K_{v^{*}} \mathcal{F}\right)=\angle\left(k_{0}^{-1} h_{1} k_{0} v, K_{v^{*}} \mathcal{F}\right)$. Notice that $K_{v^{*}}$ conjugates $\mathfrak{f}_{v^{*}}^{\perp}$ to itself, so $k_{0}^{-1} h_{1} k_{0}$ is an element in $\exp \left(\mathfrak{f}_{v^{*}}^{\perp}\right)$. In view of equation (4.1) and the fact that $k_{0}^{-1} h_{1} k_{0} v$ leaves orthogonally to $K_{v^{*}} \mathcal{F}$, we obtain $\angle\left(k_{0}^{-1} h_{1} k_{0} v, K_{v^{*}} \mathcal{F}\right) \sim\left\|k_{0}^{-1} h_{1} k_{0}\right\|=\left\|h_{1}\right\|$. Combining this estimate with equation (4.3) gives the second inequality.

Lemma 4.5. Let $X=G / K$ be a rank $r \geq 2$ irreducible symmetric space of non-compact type excluding $\mathrm{SL}(3, \mathbb{R}) / \mathrm{SO}(3)$ and $\mathrm{SL}(4, \mathbb{R}) / \mathrm{SO}(4)$, and fix a flat $\mathcal{F} \subseteq T_{x} X$ at $x$. Then there exists a constant $C>0$ that only depends on $X$, such that for any $\frac{1}{2}$-orthonormal $r$-frame $\left\{v_{1}, \ldots, v_{r}\right\}$ in $\mathcal{F}$, there is an orthonormal $(3 r-2)$-frame $\left\{v_{1}^{\prime}, v_{1}^{\prime \prime}, \ldots, v_{1}^{(r)}, v_{i}^{\prime}, v_{i}^{\prime \prime}(2 \leq i \leq r)\right\}$ in $\mathcal{F}^{\perp}$ such that

$$
\begin{gathered}
\angle\left(h v_{i}^{\prime}, \mathcal{F}^{\perp}\right) \leq C \angle\left(h v_{i}, \mathcal{F}\right) \\
\angle\left(h v_{i}^{\prime \prime}, \mathcal{F}^{\perp}\right) \leq C \angle\left(h v_{i}, \mathcal{F}\right) \\
\angle\left(h v_{1}^{(j)}, \mathcal{F}^{\perp}\right) \leq C \angle\left(h v_{1}, \mathcal{F}\right)
\end{gathered}
$$

for all $h \in K, i=2, \ldots, r, j=1, \ldots, r$.

Proof. Once we have chosen a parameter $\rho$, we will denote by $v_{i}^{*}$ a maximally singular vector in $\mathcal{F}$ that is $\rho$-close to $v_{i}$, and we will let $Q_{i}=\left(K_{v_{i}^{*}} \mathcal{F}\right)^{\perp} \simeq \bigoplus_{\alpha \in \Lambda^{+}, \alpha\left(v_{i}^{*}\right) \neq 0} \mathfrak{p}_{\alpha}$. We now fix an $\rho$ small enough so that, for every $\frac{1}{2}$-orthonormal $r$-frame $\left\{v_{1}, \ldots, v_{r}\right\} \subset \mathcal{F}$, the corresponding $\left.\left\{v_{i}^{*}\right\}\right|_{i=1} ^{r}$ are distinct. For each $v_{i}$, the vectors in $Q_{i}$ are the possible choice of vectors that satisfy the angle inequality provided by Lemma 4.4. So it suffices to find $r$ vectors in $Q_{1}$, and two vectors in each $Q_{i}(i \neq 1)$, such that the chosen $(3 r-2)$ vectors form an orthonormal frame.

Now for each root $\alpha$, we pick an orthonormal frame $\left\{b_{\alpha_{i}}\right\}$ on $\mathfrak{p}_{\alpha}$, we collect them into the set $B:=\left.\left\{b_{i}\right\}\right|_{i=1} ^{n-r}$, which forms an orthonormal frame on $\mathcal{F}^{\perp}$. We will pick the $(3 r-2)$-frame from the vectors in $B$. For instance, vector $v_{1}$ has selectable set $B_{1}:=Q_{1} \cap B$, in which we want to choose $r$ elements, while for $i=2, \ldots, r$, vector $v_{i}$ has selectable set $B_{i}:=Q_{i} \cap B$, from which we want to choose two elements. Most importantly, the $(3 r-2)$ chosen vectors have to be distinct from each other. This is a purely combinatorial problem, and can be solved by using Hall's Marriage theorem. In view of Corollary 4.3, we only need to check the cardinality condition. We notice the selectable set of $v_{i}$ is $B_{i}$ which spans $Q_{i}$, so $\left|B_{i}\right|=\operatorname{dim}\left(Q_{i}\right)$. The next lemma will estimate the dimension of the $Q_{i}$, and hence will complete the proof of Lemma 4.5 .

Lemma 4.6. Let $X=G / K$ be a rank $r \geq 2$ irreducible symmetric space of non-compact type, excluding $\mathrm{SL}(3, \mathbb{R}) / \mathrm{SO}(3)$ and $\mathrm{SL}(4, \mathbb{R}) / \mathrm{SO}(4)$, and fix a flat $\mathcal{F}$. Assume $\left\{v_{1}^{*}, \ldots, v_{r}^{*}\right\}$ spans $\mathcal{F}$, and let $Q_{i}=K_{v_{i}^{*}} \mathcal{F}$. Then for any subcollection of vectors $\left\{v_{i_{1}}^{*}, \ldots, v_{i_{k}}^{*}\right\}$, we have $\operatorname{dim}\left(Q_{i_{1}}+\ldots+Q_{i_{k}}\right) \geq(2 k+r-2)$. 
Proof. Since $Q_{i}=\left(K_{v_{i}^{*}} \mathcal{F}\right)^{\perp} \simeq \bigoplus_{\alpha \in \Lambda^{+}, \alpha\left(v_{i}^{*}\right) \neq 0} \mathfrak{p}_{\alpha}$, we obtain $Q_{i_{1}}+\ldots+Q_{i_{k}}=\bigoplus_{\alpha \in \Lambda^{+}, \alpha(V) \neq 0} \mathfrak{p}_{\alpha}$, where $V=$ $\operatorname{Span}\left(v_{i_{1}}^{*}, \ldots, v_{i_{k}}^{*}\right)$. We can estimate

$$
\operatorname{dim}\left(Q_{i_{1}}+\ldots+Q_{i_{k}}\right)=\sum_{\alpha \in \Lambda^{+}, \alpha(V) \neq 0} \operatorname{dim}\left(\mathfrak{p}_{\alpha}\right) \geq\left|\left\{\alpha \in \Lambda^{+}, \alpha(V) \neq 0\right\}\right|=\frac{1}{2}\left(|\Lambda|-\left|\left\{\alpha \in \Lambda, H_{\alpha} \in V^{\perp}\right\}\right|\right),
$$

where $V^{\perp}$ is the orthogonal complement of $V$ in $\mathcal{F}$, and $H_{\alpha}$ is the vector in $\mathcal{F}$ that represents $\alpha$.

Now we denote $t_{i}=\frac{1}{2} \max _{U \subseteq \mathcal{F}, \operatorname{dim}(U)=i}\left|\left\{\alpha \in \Lambda, H_{\alpha} \in U\right\}\right|$, the number of positive roots in the maximally rooted $i$-dimensional subspace. We use the following result that appears in the proof of [6, Lemma 5.2]. For completeness, we also add their proof here.

Claim 4.7. [6, Lemma 5.2] $t_{i}-t_{i-1} \geq i$, for $1 \leq i \leq r-1$.

Proof. This is proved by induction on $i$. For $i=1$, the inequality holds since $t_{0}=0$ and $t_{1}=1$. Assuming $t_{i-1}-t_{i-2} \geq i-1$ holds, we let $V_{i-1}$ be an $(i-1)$-dimensional maximally rooted subspace. By definition, the number of roots that lie in $V_{i-1}$ is $2 t_{i-1}$. There exists a root $\alpha$ so that $H_{\alpha}$ does not lie in $V_{i-1}$, and also does not lie on its orthogonal complement (by irreducibility of the root system). So $H_{\alpha}^{\perp} \cap V_{i-1}:=Z$ is a codimension one subspace in $V_{i-1}$. By the induction hypothesis, there are at least $i-1$ pairs of root vectors that lie in $V_{i-1}-Z$, call them $\pm H_{\alpha_{1}}, \ldots, \pm H_{\alpha_{i-1}}$. Hence by properties of root system [11, Proposition 2.9.3], either $\pm\left(H_{\alpha}+H_{\alpha_{l}}\right)$ or $\pm\left(H_{\alpha}-H_{\alpha_{l}}\right)$ is a pair of root vectors, for each $1 \leq l \leq i-1$. Along with $\pm H_{\alpha}$, these pairs of vectors lie in $\left(V_{i-1} \oplus\left\langle H_{\alpha}\right\rangle\right)-V_{i-1}$. We have now found $2 i$ root vectors in the $i$-dimensional subspace $V_{i-1} \oplus\left\langle H_{\alpha}\right\rangle$, which do not lie on the maximally rooted subspace $V_{i-1}$. This shows $t_{i}-t_{i-1} \geq i$, proving the claim.

Finally, we can estimate $\operatorname{dim}\left(Q_{i_{1}}+\ldots+Q_{i_{k}}\right) \geq \frac{1}{2}\left(|\Lambda|-\left|\left\{\alpha \in \Lambda, H_{\alpha} \in V^{\perp}\right\}\right|\right) \geq t_{r}-t_{r-k}$. Using the Claim, a telescoping sum gives us $t_{r}-t_{r-k} \geq r+(r-1)+\ldots+(r-k+1)=k(2 r-k+1) / 2$, whence the lower bound $\operatorname{dim}\left(Q_{i_{1}}+\ldots+Q_{i_{k}}\right) \geq k(2 r-k+1) / 2$. When $r \geq 4$, or $k<r=3$, or $k<r=2$, it is easy to check that $k(2 r-k+1) / 2 \geq 2 k+r-2$. This leaves the case when $r=k=3$, or $r=k=2$. When $r=k=3$, we can instead estimate $\operatorname{dim}\left(Q_{1}+Q_{2}+Q_{3}\right)=\operatorname{dim}\left(\mathcal{F}^{\perp}\right)=n-3 \geq 7=2 k+r-2$, provided $n \geq 10$, which only excludes the rank three symmetric space $\mathrm{SL}(4, \mathbb{R}) / \mathrm{SO}(4)$. A similar analysis when $r=k=2$ only excludes the rank two symmetric space $S L(3, \mathbb{R}) / S O(3)$. This completes the proof of Lemma 4.6, hence completing the proof of Lemma 4.5 .

Remark. In the rank two case, both Theorem 3.3 and Theorem 3.1 only give you statements about degree $=n$. Our Main Theorem then only gives surjectivity of comparison maps in top degree, which agrees with the result of [16], and the corresponding Jacobian estimate is consistent with [6] [7].

4.3. Proof of Theorem 3.3. We assume $k=r$ without loss of generality since otherwise we can always extend the $k$-frame to an $r$-frame that has small angle to $\mathcal{F}$. Our first step is to move the frame so as to lie in $\mathcal{F}$, while controlling the angles between the resulting vectors (so that we can apply Lemma 4.5). This is done by first moving the vectors to the respective $K_{v_{i}^{*}} \mathcal{F}$, and then moving to $\mathcal{F}$. 
As in the proof of Lemma 4.4, $\angle\left(v_{i}, \mathcal{F}\right)$ being small implies that the components of $v_{i}$ on each $\mathfrak{p}_{\alpha}$ is small. The $K$-orbit of $v_{i}$ intersects $\mathcal{F}$ finitely many times (exactly once in each Weyl chamber), and if each of these intersections is $\rho$-close to a maximally singular vector, choose $v_{i}^{*}$ to be the one closest to $v_{i}$. The element in $K$ moving $v_{i}$ to $\mathcal{F}$ will almost lie in $K_{v_{i}^{*}}$ (by an argument similar to the one in Lemma 4.4). By decomposing this element as a product $\hat{k}_{i} k_{i}$, we obtain a small $k_{i}$ which sends $v_{i}$ to $K_{v_{i}^{*}} \mathcal{F}$ (and $\hat{k}_{i} \in K_{v_{i}^{*}}$ ). If $k_{i}^{-1}=\exp \left(u_{i}\right.$ ), we have $u_{i} \in \bigoplus_{\alpha \in \Lambda^{+}, \alpha\left(v^{*}\right) \neq 0} \mathfrak{F}_{\alpha}$.

We now estimate the norm $\left\|k_{i}\right\|$. From the identification of norms in a small neighborhood of the identity, we have $\left\|k_{i}\right\|=\left\|u_{i}\right\|$. Since $\hat{k}_{i}$ is an element in $K_{v_{i}^{*}}$ that sends $k_{i} v_{i}$ to $\mathcal{F}$, an argument similar to the proof of second inequality in Lemma 4.4 gives us

$$
\angle\left(v_{i}, K_{v_{i}^{*}} \mathcal{F}\right)=\angle\left(\left(\hat{k}_{i} k_{i}^{-1} \hat{k}_{i}^{-1}\right)\left(\hat{k}_{i} k_{i} v_{i}\right), K_{v_{i}^{*}} \mathcal{F}\right) \sim_{\rho}\left\|\hat{k}_{i} k_{i}^{-1} \hat{k}_{i}^{-1}\right\|=\left\|k_{i}\right\|
$$

(where the constant will depend on the choice of $\rho$ ). On the other hand, since $\mathcal{F} \subset K_{v_{i}^{*}} \mathcal{F}$, we obtain $\angle\left(v_{i}, K_{v_{i}^{*}} \mathcal{F}\right) \leq \angle\left(v_{i}, \mathcal{F}\right)$. But by hypothesis, $\angle\left(v_{i}, \mathcal{F}\right)<\epsilon$. Putting all this together, we see that, for each fixed $\rho$, there exists a constant $C^{\prime}$ that only depends on $X$, so that each of the $\left\|k_{i}\right\|$ is bounded above by $\frac{1}{2} C^{\prime} \epsilon$. In particular, any $\left\{k_{i}\right\}_{i=1}^{r}$ perturbation of an orthonormal frame gives rise to a $C^{\prime} \epsilon$-orthonormal frame, and hence the collection $\left\{k_{1} v_{1}, \ldots, k_{r} v_{r}\right\}$ forms a $C^{\prime} \epsilon$-orthonormal frame.

Next, since $\hat{k}_{i}$ is an element in $K_{v_{i}^{*}}$, it leaves $v_{i}^{*}$ fixed. From triangle inequality we obtain

$$
\angle\left(\hat{k}_{i} k_{i} v_{i}, k_{i} v_{i}\right) \leq 2 \angle\left(k_{i} v_{i}, v_{i}^{*}\right)<2 \rho .
$$

It follows that the collection of vectors $\left\{\hat{k}_{1} k_{1} v_{1}, \ldots, \hat{k}_{r} k_{r} v_{r}\right\} \subset \mathcal{F}$ is obtained from the $C^{\prime} \epsilon$-orthonormal frame $\left\{k_{1} v_{1}, \ldots, k_{r} v_{r}\right\}$ by rotating each of the various vectors by an angle of at most $2 \rho$ hence forms a $\left(C^{\prime} \epsilon+4 \rho\right)$ orthonormal basis in $\mathcal{F}$. In particular, once $\rho$ and $\delta$ are chosen small enough, it gives us a $1 / 2$-orthonormal basis inside $\mathcal{F}$.

Applying Lemma 4.5 to the $1 / 2$-orthonormal frame $\left\{\hat{k}_{1} k_{1} v_{1}, \ldots, \hat{k}_{r} k_{r} v_{r}\right\} \subset \mathcal{F}$ gives us an orthonormal $(3 r-2)$-frame $\left\{v_{1}^{\prime}, \ldots, v_{1}^{(r)}, v_{i}^{\prime}, v_{i}^{\prime \prime}(2 \leq i \leq r)\right\}$ such that the angle inequalities hold. Now by the second inequality of Lemma 4.4, we have the following inequalities:

$$
\begin{gathered}
\angle\left(h v_{i}^{\prime}, \mathcal{F}^{\perp}\right) \leq C \angle\left(h k_{i} v_{i}, K_{v_{i}^{*}} \mathcal{F}\right) \leq C \angle\left(h k_{i} v_{i}, \mathcal{F}\right) \\
\angle\left(h v_{i}^{\prime \prime}, \mathcal{F}^{\perp}\right) \leq C \angle\left(h k_{i} v_{i}, K_{v_{i}^{*}} \mathcal{F}\right) \leq C \angle\left(h k_{i} v_{i}, \mathcal{F}\right) \\
\angle\left(h v_{1}^{(j)}, \mathcal{F}^{\perp}\right) \leq C \angle\left(h k_{1} v_{1}, K_{v_{1}^{*}} \mathcal{F}\right) \leq C \angle\left(h k_{1} v_{1}, \mathcal{F}\right)
\end{gathered}
$$

for $2 \leq i \leq r, 1 \leq j \leq r$ and any $h \in K$. Finally we translate each of the vectors $v_{i}^{\prime}$, $v_{i}^{\prime \prime}$ by $k_{i}^{-1}$, and each $v_{1}^{(j)}$ by $k_{1}^{-1}$, producing a $C^{\prime} \epsilon$-orthonormal $(3 r-2)$-frame that satisfies the inequalities in Theorem 3.3 , hence completing the proof.

\section{SURJECTIVITY OF THE COMPARISON MAP IN BOUNDED COHOMOLOGY}

In this Section, we provide some background on cohomology (see Section 5.1), establish the Main Theorem (Section 5.2), establish some limitations on our technique of proof (Section 5.3), and work out a detailed class of examples (Section 5.4). 
5.1. Bounded cohomology. Let $X=G / K$ be a symmetric space of non-compact type, and $\Gamma$ be a cocompact lattice in $G$. We recall the definition of group cohomology, working with $\mathbb{R}$ coefficients (so that we can relate these to the de Rham cohomology). Let $C^{n}(\Gamma, \mathbb{R})=\left\{f: \Gamma^{n} \rightarrow \mathbb{R}\right\}$ be the space of $n$-cochains. Then the coboundary map $d: C^{n}(\Gamma, \mathbb{R}) \rightarrow C^{n+1}(\Gamma, \mathbb{R})$ is defined by

$$
\begin{gathered}
d f\left(\gamma_{1}, \ldots, \gamma_{n+1}\right)=f\left(\gamma_{2}, \ldots, \gamma_{n+1}\right)+\sum_{i=1}^{n}(-1)^{i} f\left(\gamma_{1}, \ldots \gamma_{i-1}, \gamma_{i} \gamma_{i+1}, \gamma_{i+2}, \ldots, \gamma_{n+1}\right) \\
+(-1)^{n+1} f\left(\gamma_{1}, \ldots, \gamma_{n}\right)
\end{gathered}
$$

The homology of this chain complex is $H^{*}(\Gamma, \mathbb{R})$, the group cohomology of $\Gamma$ with $\mathbb{R}$ coefficients. Moreover, if we restrict the cochains above to bounded functions, we obtain the space of bounded $n$-cochains $C_{b}^{n}(\Gamma, \mathbb{R})=\left\{f: \Gamma^{n} \rightarrow \mathbb{R} \mid f\right.$ is bounded $\}$ and the corresponding bounded cohomology $H_{b}^{*}(\Gamma, \mathbb{R})$ of $\Gamma$. The inclusion of the bounded cochains into the ordinary cochains induces the comparison map $H_{b}^{*}(\Gamma, \mathbb{R}) \rightarrow$ $H^{*}(\Gamma, \mathbb{R})$.

Similarly, we can define the (bounded) continuous cohomology of $G$, by taking the space of continuous $n$-cochains $C_{c}^{n}(G, \mathbb{R})=\left\{f: G^{n} \rightarrow \mathbb{R} \mid f\right.$ is continuous $\}$ or the space of bounded continuous cochains $C_{c, b}^{n}(G, \mathbb{R})=\left\{f: G^{n} \rightarrow \mathbb{R} \mid f\right.$ is continuous and bounded $\}$. With the same coboundary maps as above, this gives two new chain complexes, whose homology will be denoted by $H_{c}^{*}(G, \mathbb{R})$ and $H_{c, b}^{*}(G, \mathbb{R})$ respectively. Again, one has a naturally induced comparison $\operatorname{map} H_{c, b}^{*}(G, \mathbb{R}) \rightarrow H_{c}^{*}(G, \mathbb{R})$.

Now let $M=X / \Gamma$ be the closed locally symmetric space covered by $X$. Note that $M$ is a $K(\Gamma, 1)$, so

$$
H_{d R}^{*}(M, \mathbb{R}) \simeq H_{\text {sing }}^{*}(M, \mathbb{R}) \simeq H^{*}(\Gamma, \mathbb{R})
$$

The isomorphism between the de Rham cohomology and group cohomology is explicitly given by

$$
\begin{gathered}
\phi: H_{d R}^{k}(M, \mathbb{R}) \rightarrow H^{k}(\Gamma, \mathbb{R}) \\
\omega \mapsto f_{\omega}
\end{gathered}
$$

where $f_{\omega}\left(\gamma_{1}, \ldots, \gamma_{k}\right)=\int_{\Delta\left(\gamma_{1}, \ldots, \gamma_{k}\right)} \widetilde{\omega}$. Here, $\widetilde{\omega}$ is a lift of $\omega$ to $X$, and $\Delta\left(\gamma_{1}, \ldots, \gamma_{k}\right)$ is any natural $C^{1} k$-filling with ordered vertices $\left\{x, \gamma_{1} x,\left(\gamma_{1} \gamma_{2}\right) x, \ldots,\left(\gamma_{1} \gamma_{2} \cdots \gamma_{k}\right) x\right\}$ for some fixed basepoint $x \in X$ (for instance, one can choose $\Delta\left(\gamma_{1}, \ldots, \gamma_{k}\right)$ to be the geodesic coning simplex, see Dupont [9]). Alternatively, we can use the barycentric straightened $C^{1}$ simplex $s t\left(\Delta\left(\gamma_{1}, \ldots, \gamma_{k}\right)\right.$ ) (which we defined in Section 2.3). That is to say, if we define $\overline{f_{\omega}}\left(\gamma_{1}, \ldots, \gamma_{k}\right)=\int_{s t\left(\Delta\left(\gamma_{1}, \ldots, \gamma_{k}\right)\right)} \widetilde{\omega}$, then $\overline{f_{\omega}}$ represents the same cohomology class as $f_{\omega}$. This is due to the fact that the barycentric straightening is $\Gamma$-equivariant (see [16, Section 3.2]). We call $\overline{f_{\omega}}$ the barycentrically straightened cocycle.

On the other hand, there is a theorem of van Est [18] which gives the isomorphism between the relative Lie algebra cohomology $H^{*}(\mathfrak{g}, \mathfrak{f}, \mathbb{R})$ and the continuous bounded cohomology $H_{c}^{*}(G, \mathbb{R})$. A class in $H^{k}(\mathfrak{g}, \mathfrak{f}, \mathbb{R})$ can be expressed by an alternating $k$-form $\varphi$ on $\mathfrak{g} / \mathfrak{f} \simeq T_{x} X$. By left translation, it gives a closed $C^{\infty} k$-form $\widetilde{\varphi}$ on $X=G / K$. In [9], this isomorphism is explicitly given by

$$
\phi: H^{k}(\mathfrak{g}, \mathfrak{f}, \mathbb{R}) \rightarrow H_{c}^{k}(G, \mathbb{R})
$$




$$
\varphi \mapsto f_{\varphi}
$$

where $f_{\varphi}\left(g_{1}, \ldots, g_{k}\right)=\int_{\Delta\left(g_{1}, \ldots, g_{k}\right)} \widetilde{\varphi}$, and $\Delta\left(g_{1}, \ldots, g_{k}\right)$ is the geodesic simplex with ordered vertices consisting of $\left\{x, g_{1} x,\left(g_{1} g_{2}\right) x, \ldots,\left(g_{1} g_{2} \cdots g_{k}\right) x\right\}$ for some fixed basepoint $x \in X$. Again, we can replace $\Delta\left(g_{1}, \ldots, g_{k}\right)$ by the barycentric straightened $C^{1} \operatorname{simplex} s t\left(\Delta\left(g_{1}, \ldots, g_{k}\right)\right)$, and the resulting barycentrically straightened function $\overline{f_{\varphi}}\left(g_{1}, \ldots, g_{k}\right)=\int_{s t\left(\Delta\left(g_{1}, \ldots, g_{k}\right)\right)} \widetilde{\varphi}$ is in the same cohomology class as $f_{\varphi}$.

5.2. Proof of the Main Theorem. In this section, we use Theorem 3.1 to establish the Main Theorem. We need to show both comparison maps $\eta$ and $\eta^{\prime}$ are surjective. Let us start with $\eta$. We use the van Est isomorphism (see Section 5.1 ) to identify $H_{c}^{*}(G, \mathbb{R})$ with $H^{*}(\mathfrak{g}, \mathfrak{f}, \mathbb{R})$. For any class $\left[f_{\varphi}\right] \in H_{c}^{k}(G, \mathbb{R})$ where $f_{\varphi}\left(g_{1}, \ldots, g_{k}\right)=\int_{\Delta\left(g_{1}, \ldots, g_{k}\right)} \widetilde{\varphi}$, we instead choose the barycentrically straightened representative $\overline{f_{\varphi}}$. Then for any $\left(g_{1}, \ldots, g_{k}\right) \in G^{k}$, we have

$$
\left|\overline{f_{\varphi}}\left(g_{1}, \ldots, g_{k}\right)\right|=\left|\int_{s t\left(\Delta\left(g_{1}, \ldots, g_{k}\right)\right)} \widetilde{\varphi}\right| \leq\left|\int_{\Delta_{s}^{k}} s t_{V}^{*} \widetilde{\varphi}\right| \leq \int_{\Delta_{s}^{k}}\left|\operatorname{Jac}\left(s t_{V}\right)\right| \cdot\|\widetilde{\varphi}\| d \mu_{0}
$$

where $d \mu_{0}$ is the standard volume form of $\Delta_{s}^{k}$. But from Proposition 2.3 and Theorem 3.1, the expression $\left|\operatorname{Jac}\left(s t_{V}\right)\right|$ is uniformly bounded above by a constant (independent of the choice of vertices $V$ and the point $\delta \in \Delta_{s}^{k}$ ), while the form $\widetilde{\varphi}$ is invariant under the $G$-action, hence bounded in norm. It follows that the last expression above is less than some constant $C$ that depends only on the choice of alternating form $\varphi$. We have thus produced, for each class $\left[f_{\varphi}\right]$ in $H_{c}^{k}(G, \mathbb{R})$, a bounded representative $\overline{f_{\varphi}}$. So the comparison map $\eta$ is surjective. The argument for surjectivity of $\eta^{\prime}$ is virtually identical, using the explicit isomorphism between $H^{k}(\Gamma, \mathbb{R})$ and $H_{d R}^{k}(M, \mathbb{R})$ discussed in Section 5.1 For any class $\left[f_{\omega}\right] \in H^{k}(\Gamma, \mathbb{R})$, we choose the barycentrically straightened representative $\overline{f_{\omega}}$. The differential form $\widetilde{\omega}$ has bounded norm, as it is the $\Gamma$ invariant lift of the smooth differential form $\omega$ on the compact manifold $M$. So again, the estimate in Equation (5.1) shows the representative $\overline{f_{\omega}}$ is bounded, completing the proof.

5.3. Obstruction to Straightening Methods. In this section, we give a general obstruction to the straightening method that is applied in section 5.2. In the next section, we will use this to give some concrete examples showing that Theorem 3.1 is not true when $\operatorname{dim}(S) \leq n-r$. Throughout this section, we let $X=G / K$ be an $n$-dimensional symmetric space of non-compact type, and we give the following definitions.

Definition 5.1. Let $C^{0}\left(\Delta^{k}, X\right)$ be the set of singular $k$-simplices in $X$, where $\Delta^{k}$ is assumed to be equipped with a fixed Riemannian metric. Assume that we are given a collection of maps $s t_{k}: C^{0}\left(\Delta^{k}, X\right) \rightarrow C^{0}\left(\Delta^{k}, X\right)$. We say this collection of maps forms a straightening if it satisfies the following properties:

(a) the maps induces a chain map, that is, it commutes with the boundary operators.

(b) $s t_{n}$ is $C^{1}$ smooth, that is, the image of $\operatorname{st}_{n}$ lies in $C^{1}\left(\Delta^{n}, X\right)$.

For a subgroup $H \leq G$, we say the straightening is $H$-equivariant if the maps st $t_{k}$ all commute with the H-action.

Since $X$ is simply connected, property (a) of Definition 5.1 implies that the chain map $s t_{*}$ is actually chain homotopic to the identity. Also, property (b) of Definition 5.1 implies the image of any straightened 
$k$-simplex is $C^{1}$-smooth, i.e. $\operatorname{Im}\left(s t_{k}\right) \subset C^{1}\left(\Delta^{k}, X\right)$. The barycentric straightening introduced in Section 2.3 is a $G$-equivariant straightening. As we saw in Section 5.2, obtaining a uniform control on the Jacobian of the straightened $k$-simplices immediately implies a surjectivity result for the comparison map from bounded cohomology to ordinary cohomology. This motivates the following:

Definition 5.2. We say the straightening is $k$-bounded, if there exists a constant $C>0$, depending only on $X$ and the chosen Riemannian metric on $\Delta^{k}$, with the following property. For any $k$-dimensional singular simplex $f \in C^{0}\left(\Delta^{k}, X\right)$, and corresponding straightened simplex $s t_{k}(f): \Delta^{k} \rightarrow X$, the Jacobian of $\operatorname{st}_{k}(f)$ satisfies:

$$
\left|\operatorname{Jac}\left(s t_{k}(f)\right)(\delta)\right| \leq C
$$

where $\delta \in \Delta^{k}$ is arbitrary (and the Jacobian is computed relative to the fixed Riemannian metric on $\Delta^{k}$ ).

Our Theorem 3.1 and Proposition 2.3 then tells us that, when $r=\mathbb{R}-\operatorname{rank}(G) \geq 2$ (excluding the two cases $\mathrm{SL}(3, \mathbb{R}) / \mathrm{SO}(3)$ and $\mathrm{SL}(4, \mathbb{R}) / \mathrm{SO}(4))$, our barycentric straightening is $k$-bounded for all $k \geq n-r+2$. One can wonder whether this range can be improved. In order to obtain obstructions, we recall [16, Theorem 2.4]. Restricting to the case of locally symmetric spaces of non-compact type, the theorem says:

Theorem 5.3. [16, Theorem 2.4] Let $M$ be an n-dimensional locally symmetric space of non-compact type, with universal cover $X$, and $\Gamma$ be the fundamental group of $M$. If $X$ admits an $n$-bounded, $\Gamma$-equivariant straightening, then the simplicial volume of $M$ is positive.

Corollary 5.4. If $X$ splits off an isometric $\mathbb{R}$-factor, then $X$ does not admit an n-bounded, G-equivariant straightening.

Proof. Let $X \simeq X_{0} \times \mathbb{R}$ for some symmetric space $X_{0}$. If $X$ admits an $n$-bounded, $G$-equivariant straightening, then consider a closed manifold $M \simeq M_{0} \times S^{1}$, where $\widetilde{M}_{0} \simeq X_{0}$. According to Theorem 5.3, the simplicial volume $\|M\|$ is positive. But on the other hand $\|M\|=\left\|M_{0} \times S^{1}\right\| \leq C \cdot\left\|M_{0}\right\| \cdot\left\|S^{1}\right\|=0$. This contradiction completes the proof.

We will use subspaces satisfying Corollary 5.4 to obstruct bounded straightenings.

Definition 5.5. For $X$ a symmetric space of non-compact type, we define the splitting rank of $X$, denoted $\operatorname{srk}(X)$, to be the maximal dimension of a totally geodesic submanifold $Y \subset X$ which splits off an isometric $\mathbb{R}$-factor.

For the irreducible symmetric spaces of non-compact type, computations of the splitting rank can be found in a recent paper by the second author [19] (see also Berndt and Olmos [2] for some related work).

Theorem 5.6. If $k=\operatorname{srk}(X)$, then $X$ does not admit any $k$-bounded, $G$-equivariant straightening.

Proof. We show this by contradiction. Assume $X=G / K$ admits a $k$-bounded, $G$-equivariant straightening $s t_{i}$, and let $Y \subset X$ be a $k$-dimensional totally geodesic subspace which splits isometrically as $Y^{\prime} \times \mathbb{R}$. Denote by $p: X \rightarrow Y$ the orthogonal projection from $X$ to $Y$, and note that the composition $p \circ s t_{*}$ is a straightening 
on $Y$, which we denote by $\overline{s t_{*}}$. Notice $Y$ is also a symmetric space and can be identified with $G_{0} / K_{0}$, for some $G_{0}<G$, and $K_{0}<K$. Then the straightening $\overline{s t_{*}}$ is certainly $G_{0}$-equivariant. We claim it is also $k$-bounded. This is because the projection map $p$ is volume-decreasing, hence

$$
\left|J a c\left(\overline{s t_{k}}(f)\right)\right|=\left|\operatorname{Jac}\left(p\left(s t_{k}(f)\right)\right)\right| \leq\left|\operatorname{Jac}\left(s t_{k}(f)\right)\right| \leq C
$$

for any $f \in C^{0}\left(\Delta^{k}, X\right)$. Therefore, we conclude that $Y$ admits a $G_{0}$-equivariant, $k$-bounded straightening. This contradicts Corollary 5.4

Remark. In view of Proposition 2.3 and the arguments in Section 5.2, we can view Theorem 5.6 as obstructing the bounded ratio Theorem 3.1. Specifically, if $k=\operatorname{srk}(X)$, then Theorem 5.6 tells us that one has a sequence $f_{i}: \Delta_{s}^{k} \rightarrow X$ with the property that the Jacobian of $s t_{k}\left(f_{i}\right)$ is unbounded. From the definition of our straightening maps $s t_{k}$, this means one has a sequence $V_{i}=\left\{v_{0}^{(i)}, \ldots v_{k}^{(i)}\right\} \subset X$ of $(k+1)$-tuples of points (the vertices of the singular simplices $\left.f_{i}\right)$, and a sequence of points $\delta_{i}=\left(a_{0}^{(i)}, \ldots, a_{k}^{(i)}\right)$ inside the spherical simplex $\Delta_{s}^{k} \subset \mathbb{R}^{k+1}$, satisfying the following property. If one looks at the corresponding sequence of points

$$
p_{i}:=\left(s t_{k}\left(f_{i}\right)\right)\left(\delta_{i}\right)=\operatorname{Bar}\left(\sum_{j=0}^{k} a_{j}^{(i)} \mu\left(v_{j}^{(i)}\right)\right),
$$

one has a sequence of $k$-dimensional subspace $S_{i} \subset T_{p_{i}} X$ (given by the tangent spaces $D\left(s t_{V_{i}}\right)\left(T_{\delta_{i}} \Delta_{s}^{k}\right.$ ) to the straightened simplex $s t_{k}\left(f_{i}\right)$ at the point $\left.p_{i}\right)$, and the sequence of $\operatorname{ratios} \operatorname{det}\left(\left.Q_{1}\right|_{S_{i}}\right)^{1 / 2} / \operatorname{det}\left(\left.Q_{2}\right|_{S_{i}}\right)$ tends to infinity. It is not too hard to see that, for each dimension $k^{\prime} \leq k$, one can find a $k^{\prime}$-dimensional subspace $\bar{S}_{i} \subset S_{i}$ such that the sequence of ratios of determinants, for the quadratic forms restricted to the $\bar{S}_{i}$, must also tend to infinity. Thus the bounded ratio Theorem 3.1 fails whenever $k^{\prime} \leq \operatorname{srk}(X)$.

5.4. The case of $S L(m, \mathbb{R})$. We conclude our paper with a detailed discussion of the special case of the Lie group $G=S L(m, \mathbb{R}), m \geq 5$. The continuous cohomology has been computed (see e.g. [12, pg. 299]) and can be described as follows. If $m=2 k$ is even, then $H_{c}^{*}(S L(2 k, \mathbb{R}))$ is an exterior algebra in $k$ generators in degrees $5,9, \ldots, 4 k-3,2 k$. If $m=2 k+1$ is even, then $H_{c}^{*}(S L(2 k+1, \mathbb{R}))$ is an exterior algebra in $k$ generators in degrees $5,9, \ldots, 4 k+1$.

The associated symmetric space is $X=S L(m, \mathbb{R}) / S O(m)$, and we have that

$$
n=\operatorname{dim}(X)=\operatorname{dim}(S L(m, \mathbb{R}))-\operatorname{dim}(S O(m))=\left(m^{2}-1\right)-\frac{1}{2} m(m-1)=\left(\begin{array}{c}
m+1 \\
2
\end{array}\right)-1,
$$

while the rank of the symmetric space is clearly $r=m-1$. Thus, our Main Theorem tells us that, for these Lie groups, the comparison map

$$
H_{c, b}^{*}(S L(m, \mathbb{R})) \rightarrow H_{c}^{*}(S L(m, \mathbb{R}))
$$

is surjective within the range of degrees $* \geq\left(\begin{array}{c}m+1 \\ 2\end{array}\right)-m+2$.

Observe that the exterior product of all the generators $H_{c}^{*}(S L(m, \mathbb{R}))$ yields the generator for the topdimensional cohomology, which lies in degree $\left(\begin{array}{c}m+1 \\ 2\end{array}\right)-1$. Dropping off the 5-dimensional generator in the exterior product yields a non-trivial class in degree $\left(\begin{array}{c}m+1 \\ 2\end{array}\right)-6$. Comparing with the surjectivity range in 
our Main Theorem, we see that the first interesting example occurs in the case of $S L(8, \mathbb{R})$, where our results imply that $H_{c, b}^{30}(S L(8, \mathbb{R})) \neq 0$ (as well as $H_{c, b}^{35}(S L(8, \mathbb{R})) \neq 0$, which was previously known). Of course, as $m$ increases, our method provides more and more non-trivial bounded cohomology classes. For example, once we reach $S L(12, \mathbb{R})$, we get new non-trivial bounded cohomology classes in $H_{c, b}^{68}(S L(12, \mathbb{R}))$ and $H_{c, b}^{72}(S L(12, \mathbb{R}))$.

Finally, let us consider Theorem 5.6 in the special case of $X=S L(m, \mathbb{R}) / S O(m)$. Choose a maximally singular direction in the symmetric space $X$, and let $X_{0}$ be the set of geodesics that are parallel to that direction. Without loss of generality, we can take $X_{0}=G_{0} / K_{0}$, where

$$
G_{0}=\left\{\left[\begin{array}{cc}
A & 0 \\
0 & a
\end{array}\right] \mid \operatorname{det}(A) \cdot a=1, a>0\right\}
$$

and $K_{0}=\mathrm{SO}(m) \cap G_{0}$. Moreover, $X_{0}$ clearly splits off an isometric $\mathbb{R}$-factor, and can be isometrically identified with $\operatorname{SL}(m-1, \mathbb{R}) / \operatorname{SO}(m-1) \times \mathbb{R}$. This is the maximal dimensional subspace of $S L(m, \mathbb{R})$ that splits off an isometric $\mathbb{R}$-factor (see [2, Table 3]), and the splitting rank is just $\operatorname{dim}\left(X_{0}\right)=\left(\begin{array}{c}m \\ 2\end{array}\right)$. So in this special case, Theorem 5.6 tells us that our method for obtaining bounded cohomology classes fails once we reach degrees $\leq\left(\begin{array}{c}m \\ 2\end{array}\right)$. Comparing this to the range where our method works, we see that, in the special case where $G=S L(m, \mathbb{R})$, the only degree which remains unclear is $\left(\begin{array}{c}m \\ 2\end{array}\right)+1$. This example shows our Main Theorem is very close to the optimal possible.

\section{Concluding Remarks}

As we have seen, the technique used in our Main Theorem seems close to optimal, at least when restricted to the Lie groups $S L(m, \mathbb{R})$. Nevertheless, the authors believe that for other families of symmetric spaces, there are likely to be improvements on the range of dimensions in which a barycentric straightening is bounded.

We also note that it might still be possible to bypass the limitations provided by the splitting rank. Indeed, the splitting rank arguments show that the barycentric straightening is not $k$-bounded, when $k=\operatorname{srk}(X)$. But the barycentric straightening might still be $k^{\prime}$-bounded for some $k^{\prime}<\operatorname{srk}(X)$ (even though the bounded Jacobian Theorem 3.1 must fail for $k^{\prime}$-dimensional subspaces).

\section{REFERENCES}

[1] P. Albuquerque. Patterson-Sullivan theory in higher rank symmetric spaces. Geom. Funct. Anal., 9(1):1-28, 1999.

[2] J. Berndt and C. Olmos. Maximal totally geodesic submanifolds and index of symmetric spaces. J. Diff. Geom., 104(2):187$217,2016$.

[3] G. Besson, G. Courtois, and S. Gallot. Minimal entropy and Mostow's rigidity theorems. Ergodic Theory Dynam. Systems, 16(4):623-649, 1996.

[4] M. Burger, A. Iozzi, N. Monod, and A. Wienhard. Bounds for cohomology classes. Enseign. Math., 54:52-54, 2008.

[5] J.-L. Clerc and B. Ørsted. The Gromov norm of the Kaehler class and the Maslov index. Asian J. Math., 7(2):269-295, 2003.

[6] C. Connell and B. Farb. The degree theorem in higher rank. J. Differential Geom., 65(1):19-59, 2003.

[7] C. Connell and B. Farb. Erratum for "The degree theorem in higher rank". J. Differential Geom., 105(1):21-32, 2017. 
[8] A. Domic and D. Toledo. The Gromov norm of the Kaehler class of symmetric domains. Math. Ann., 276(3):425-432, 1987.

[9] J. L. Dupont. Simplicial de Rham cohomology and characteristic classes of flat bundles. Topology, 15(3):233-245, 1976.

[10] J. L. Dupont. Bounds for characteristic numbers of flat bundles. In Algebraic topology, Aarhus 1978 (Proc. Sympos., Univ. Aarhus, Aarhus, 1978), volume 763 of Lecture Notes in Math., pages 109-119. Springer, Berlin, 1979.

[11] P. B. Eberlein. Geometry of nonpositively curved manifolds. Chicago Lectures in Mathematics. University of Chicago Press, Chicago, IL, 1996.

[12] D. B. Fuks. Cohomology of Infinite-Dimensional Lie Algebras. Springer-Verlag, 1986.

[13] M. Gromov. Volume and bounded cohomology. Inst. Hautes Études Sci. Publ. Math., 56:5-99, 1982.

[14] T. Hartnick and A. Ott. Surjectivity of the comparison map in bounded cohomology for Hermitian Lie groups. Int. Math. Res. Not. IMRN, 9:2068-2093, 2012.

[15] I. Kim and S. Kim. Simplicial volume, barycenter method, and bounded cohomology. http://arxiv.org/abs/1503.02381, preprint, 2015.

[16] J.-F. Lafont and B. Schmidt. Simplicial volume of closed locally symmetric spaces of non-compact type. Acta Math., 197(1):129-143, 2006.

[17] N. Monod. An invitation to bounded cohomology. In International Congress of Mathematicians. Vol. II, pages 1183-1211. Eur. Math. Soc., Zürich, 2006.

[18] W. T. van Est. On the algebraic cohomology concepts in Lie groups. I, II. Nederl. Akad. Wetensch. Proc. Ser. A. $58=$ Indag. Math., 17:225-233, 286-294, 1955.

[19] S. Wang. On splitting rank of non-compact type symmetric spaces and bounded cohomology. http://arxiv.org/abs/1602.01495. preprint to appear in J. Topol. Anal., 2016.

Department of Mathematics, The Ohio State University, 231 W. 18th Ave., Columbus, OH 43210, U.S.A.

E-mail address: jlafont@math.ohio-state.edu

Department of Mathematics, Indiana University, 831 E. Third St., Bloomington, IN 47405, U.S.A.

E-mail address: wang679@iu.edu 